\title{
Analysis of The Impact of Diabetes on The Dynamical Transmission of Tuberculosis
}

\author{
D.P. Moualeu ${ }^{1,4}$, S. Bowong ${ }^{2,4,5, *}$, J.J. Tewa ${ }^{3,4}$, Y. Emvudu ${ }^{1}$ \\ ${ }^{1}$ Department of Mathematics, Faculty of Science, University of Yaounde I, PO Box 812 Yaounde \\ Cameroon \\ ${ }^{2}$ Laboratory of Applied Mathematics, Department of Mathematics and Computer Science, Faculty of \\ Science, University of Douala, PO Box 24157 Douala, Cameroon \\ 3 Department of Mathematics and Physics, National Advanced School of Engineering (Polytechnic) \\ University of Yaounde I, PO Box 8390 Yaounde, Cameroon \\ ${ }^{4}$ UMI 209 IRD/UPMC UMMISCO, Bondy-France and Project GRIMCAPE, LIRIMA, University of \\ Yaounde I, Cameroon \\ ${ }^{5}$ The Abdus Salam International Centre for Theoretical Physics, PO Box 538, Strada Costiera 11 \\ I-34014 Trieste, Italy
}

\begin{abstract}
Tuberculosis (TB) remains a major global health problem. A possible risk factor for TB is diabetes (DM), which is predicted to increase dramatically over the next two decades, particularly in low and middle income countries, where TB is widespread. This study aimed to assess the strength of the association between TB and DM. We present a deterministic model for TB in a community in order to determine the impact of DM in the spread of the disease. The important mathematical features of the TB model are thoroughly investigated. The epidemic threshold known as the basic reproduction number and equilibria for the model are determined and stabilities analyzed. The model is numerically analyzed to assess the impact of DM on the transmission dynamics of TB. We perform sensitivity analysis on the key parameters that drive the disease dynamics in order to determine their relative importance to disease transmission and prevalence. Numerical simulations suggest that DM enhances the TB transmission and progression to active TB in a community. The results suggest that there is a need for increased attention to intervention strategies such as the chemoprophylaxis of TB latent individuals and treatment of active TB in people with DM, which may include testing for suspected diabetes, improved glucose control, and increased clinical and therapeutic monitoring in order to reduce the burden of the disease.
\end{abstract}

Keywords and phrases: nonlinear dynamical systems, epidemiological models, diabetes, tuberculosis, stability, bifurcation

Mathematics Subject Classification: 34A34, 34D23, 34D40, 92D30

\footnotetext{
* Corresponding author. E-mail: sbowong@gmail.com
} 


\section{Introduction}

Despite the availability of effective therapy, tuberculosis (TB) continues to infect an estimated onethird of the world's population, to cause disease in 8.8 million people per year, and to kill 2 million of those afflicted [1]. Current TB control measures focus on the prompt detection and treatment of those with infectious forms of the disease to prevent further transmission of the organism. Despite the enormous success of this strategy in TB control, the persistence of TB in many parts of the world suggests the need to expand control efforts to identify and address the individual and social determinants of the disease. It is estimated that between the years 2000 and 2020, about one billion people will be newly infected, 200 million will become sick, and 35 million people will die from TB worldwide if TB control is not further strengthened [1]. Nearly $80 \%$ of both incident cases and deaths will be in 22 "high-burden" countries, mostly located in sub-Saharan Africa and Southeast of Asia, where the problem is exacerbated by high rates of co-infection with HIV [1]. Most individuals infected with TB develop an asymptomatic latent infection. Over the course of a lifetime, approximately $5-10 \%$ of latent infections progress to active TB in otherwise healthy individuals, either by endogenous reactivation of the existing infection or exogenous re-infection with a new strain. The probability to progress to active TB is markedly higher for people who are infected by HIV (up to $10 \%$ per year). Although most countries with a high burden of TB have adopted and widely implemented the World Health Organization's Stop TB Strategy, the rate of decline in case numbers has been slower than expected [1,2]. Possible explanations include patient and health system delays in diagnosis and treatment, and the rise of risk factors including co-infections (notably with human immunodeficiency virus, HIV), air pollution, alcohol abuse, crowding, diabetes, malnutrition, tobacco smoking and urbanization [3].

Diabetes is one of the major causes of premature illness and death worldwide. Non-communicable diseases including diabetes account for $60 \%$ of all deaths worldwide. Diabetes is a chronic disease that occurs either when the pancreas does not produce enough insulin or when the body cannot effectively use the insulin it produces. Insulin is a hormone that regulates blood sugar. Hyperglycaemia, or raised blood sugar, is a common effect of uncontrolled diabetes and over time leads to serious damage to many of the body's systems, especially the nerves and blood vessels. There exists two types of diabetes [4]. Type 1 diabetes (previously known as insulin-dependent, juvenile or childhood-onset) is characterized by a deficient insulin production and requires daily administration of insulin. The cause of type 1 diabetes is not known and it is not preventable with current knowledge. Symptoms include excessive excretion of urine (polyuria), thirst (polydipsia), constant hunger, weight loss, vision changes and fatigue. These symptoms may occur suddenly. Type 2 diabetes (formerly called non-insulin-dependent or adult-onset) results from the body's ineffective use of insulin. Type 2 diabetes comprises $90 \%$ of people with diabetes around the world, and is largely the result of excess body weight and physical inactivity. Symptoms may be similar to those of type 1 diabetes, but are often less marked. As a result, the disease may be diagnosed several years after onset, once complications have already arisen. WHO predicts that developing countries will bear the brunt of this epidemic in the 21 st century. Currently, more than $70 \%$ of people with diabetes live in low and middle income countries. An estimated 285 million people, corresponding to $6.4 \%$ of the world's adult population, have lived with diabetes in 2010 [4]. The number is expected to grow to 438 million by 2030 , corresponding to $7.8 \%$ of the adult population. While the global prevalence of diabetes is $6.4 \%$, the prevalence varies from $10.2 \%$ in the Western Pacific to $3.8 \%$ in the African region. However, the African region is expected to experience the highest increase.

More recently, multiple rigorous epidemiological studies have demonstrated that DM is positively associated with TB. It has been evident for decades that there is a strong association between DM use and risk of TB. The current diabetes epidemic may lead to a resurgence of TB in endemic regions, especially in urban areas. This potentially carries a risk of global spread with serious implications for TB control and the achievement of the United Nations Millennium Development Goals. The World Health Organization suspects that TB control is being undermined by the growing number of patients with DM in the world $[7,8]$. Prior to the 1950's reports of an association between diabetes (primarily Type 1) and TB were frequent in the literature, but they waned as insulin and drugs against TB became available 
$[9,10]$. This association (now with type 2 diabetes) was recognized again in the 1990's [11-14] and is currently supported by a growing body of literature [7-16]. According to a recent meta-analysis, diabetes patients have three times the risk of contracting TB as non-diabetics [16] and studies report the fraction of TB cases attributable to diabetes to be between $15 \%$ and $25 \%$. Studies suggest that diabetes depresses the immune response, which in turn facilitates infection with Mycobacterium tuberculosis (MTB) and/or progression to symptomatic disease. This is corroborated by the fact that DM is generally diagnosed before TB develops [16]. A probable cause of increased incidence of pulmonary tuberculosis in diabetics could be the defect in host defenses and immune cell functions $[17,18]$. The immune derangements predominantly involve the cell-mediated arm of the immune system. Also, the degree of hyperglycemia has been found to have a distinct influence on the microbicidal function of macrophages, with even brief exposures to blood sugar level of $200 \mathrm{mg}$ significantly depressing the respiratory burst of these cells $[23,24]$. This is borne out by the observation that in poorly controlled diabetics, with high levels of glycated haemoglobin, TB follows a more destructive course and is associated with higher mortality. Multiple pulmonary physiologic abnormalities have also been documented in diabetics that contribute to delayed clearance of and spread of infection in the host [17]. Infection with tubercle bacilli leads to further alterations in cytokines, monocyte-macrophages and CD4/CD8 T cell populations $[19,20]$. The balance of the T lymphocyte subsets CD4 and CD8 plays a central role in the modulation of host defenses against mycobacteria and has a profound influence on the rate of regression of active pulmonary tuberculosis $[21,22]$.

However, in reviewing and summarising the published work on the complex relation between tuberculosis and DM and their respective treatments, we have found that many important topics have been poorly studied or not studied at all. Although tuberculosis is clearly more common in diabetic patients, several questions remain unanswered that would greatly affect the clinical management of the two diseases and, thus, merit increased attention: does DM leads to increased susceptibility to initial tuberculosis infection, or, rather, does DM leads to increased progression from latent TB to active TB? Would screening for and treatment of latent tuberculosis in diabetic patients be appropriate and cost-effective; if so, in which populations? Which tuberculosis patients should we screen for DM? Does diabetes substantially prolong sputum smear and culture positivity; if so, are diabetic patients at higher risk of relapse than non-diabetic patients, and might this affect appropriate treatment duration? Does aggressive management of DM in patients with tuberculosis affect treatment outcomes? If mortality is higher in TB patients with diabetes, what are the most common preventable causes of death in coaffected individuals? Is there a relation between low rifampicin concentrations and TB treatment failure or acquisition of resistance in diabetic patients; if so, what might be the role of therapeutic drug monitoring?

There is a need for a strong qualitative assessment of the population-level implication of the consequences due to the interaction between TB and DM. The main interest is to understand the long and short term behavior of the effects of DM on the dynamics of TB and to predict whether the diseases will die out or will persist. A brief survey on previous works provides the context of this paper. Using data from India, Stevenson et al. [23] have illustrated that DM makes a substantial contribution to TB incidence. They found that the current diabetes epidemic may lead to a resurgence of TB in endemic regions, especially in urban areas. Murray and Jeon [24] have studied the relationship between DM and TB. These studies consisted of over 1.7 million participants who had 17,698 cases of TB. The aggregated data revealed a three-fold increase in risk of active TB for patients with DM. They suggested that TB controls programs should consider targeting patients with DM for interventions such as active case finding and the treatment of latent TB and, conversely, that efforts to diagnose, detect, and treat DM may have a beneficial impact on TB control. Dye et al. [25] have shown quantitatively how five specific, interacting, developmental factors: population growth, aging, body mass index, diabetes and urbanization, are working for and against contemporary TB control programmes in two contrasting Asian countries. In their paper, Dooley and Chaisson [26] have reviewed the epidemiology of TB and DM epidemics, and provide a synopsis of the evidence for the role of DM in susceptibility to, clinical presentation of, and response to treatment for TB. On the other hand, the global and national response to the HIV-associated 
TB epidemic was slow and uncoordinated. The first reports of the association between HIV/AIDS and TB were published in the late 1980s, and warnings were clearly articulated in the early 1990s about the impending scale of the forthcoming dual epidemic. Little action was taken, and HIV and TB programmes barely interacted at global, national or district levels. It was not until 2004, with the release of the WHO interim policy, that collaborative HIV and TB activities got under way and progress was made in the fight against the two diseases. So, it would be useful to not make the same mistake with DM and TB. In fact, if we are forewarned and prepared we stand a better chance of reducing the dual burden of disease of DM and TB than was the case with HIV and TB.

Various theoretical studies have been carried out on the mathematical modelling of TB transmission focusing on a number of different issues [27-32]. None of these studies has considered the effect of DM on the transmission dynamics of TB. Since mathematical models on the effect of DM in the spread of TB are lacking, it is therefore the intention of this study to investigate the impact of DM on the transmission dynamics of TB in the community. The model incorporates some key epidemiological features of TB such as exogenous reinfections, chemoprophylaxis and treatment. The main objective in this study is to forecast future trends in the incidence of TB and also to quantify the association between DM and TB in the community.

\section{The model}

\subsection{Details and explanation of the model}

The proposed model classifies the human population based on their diabetic status and TB. We assume that a non-diabetic person becomes a potential diabetic. As a first step, we assume random mixing of the population, even though diabetics and non-diabetics may well have different mixing patterns. The model sub-divides the human population into the following classes: susceptible individuals who are potential diabetics $\left(S_{1}\right)$, latently infected individuals who are potential diabetics $\left(E_{1}\right)$, infectious who are potential diabetics $\left(I_{1}\right)$, recovered individuals who are potential diabetics $\left(R_{1}\right)$, diabetics susceptible $\left(S_{2}\right)$, diabetics latently infected individuals $\left(E_{2}\right)$, diabetics infectious $\left(I_{2}\right)$, and diabetics recovered $\left(R_{2}\right)$. Thus, the total human population at time $t$ is

$$
N(t)=S_{1}(t)+S_{2}(t)+E_{1}(t)+E_{2}(t)+I_{1}(t)+I_{2}(t)+R_{1}(t)+R_{2}(t) .
$$

As a first step, we assume random mixing of the population, even though individuals with and without diabetes may well have different mixing patterns. The potential effect of non-random mixing patterns will be addressed in the near future.

Transmission of MTB occurs due to adequate contacts between susceptible individuals and infectious. Then, susceptible individuals acquire TB infection from individuals with active TB at rate $\lambda$, given by

$$
\lambda=\beta \frac{I_{1}+\varepsilon I_{2}}{N},
$$

where $\beta$ is the effective contact rate of diabetics and non-diabetics infectious that is sufficient to transmit infection to diabetics and non-diabetics susceptible individuals. Further, the modification parameter $\varepsilon>1$ accounts for the high infectiousness of diabetics infectious individuals (those in the $I_{2}$ class) in comparison to non-diabetics infectious individuals (those in the $I_{1}$ class).

Non-diabetics susceptible individuals are recruited through birth at rate $\Lambda$. The transition between these sub-populations proceeds in such a way that a susceptible individual acquires the bacteria through a contact with an infectious subject with the transmission rate $\beta$.

Non-diabetics susceptible individuals are infected with MTB at rate $\lambda$. A proportion $p_{1}$ of newly infected individuals who are non-diabetics develops a fast TB (development of an active TB within the first five years following the infection) and the complementary part $\left(1-p_{1}\right)$ becomes latently infected and enters the $E_{1}$ class. Once latently infected, an individual can follow a chemoprophylaxis. We assume 
that the chemoprophylaxis of latently infected individuals reduces their reactivation. We denote by $r_{1}$ the rate of chemoprophylaxis of non-diabetics latently-infected individuals. Thus, $r_{1} E_{1}$ is the number of nondiabetics latently-infected individuals who received chemoprophylaxis. Non-diabetics latently infected individuals who does not received effective chemoprophylaxis develop an active TB through endogenous reactivation (most people have tuberculosis which can be activated in situations of DM which turns off the production of cell-mediated immunity in the lungs, rendering the patient more susceptible to the development of progressive disease from the latent TB infection, $[17,18]$ and exogenous reinfections at rates $k_{1}\left(1-r_{1}\right)$ and $\sigma_{1}\left(1-r_{1}\right) \lambda$, respectively, with $\sigma_{1} \in(0,1)$ since primary infection confers some degree of immunity (see [33], and the references therein). Secondary tuberculosis may follow reinfection or endogenous reactivation and knowledge about the relative importance of these mechanisms in specific communities are important possible drivers of the disease dynamics. Once in the active stage of the disease and after receiving an effective therapy, a non-diabetics infectious may spontaneously recover from the disease at rate $\gamma_{1}$, and move to the recovered class $R_{1}$ (though there may contain some live bacilli). As suggested by Styblo [34], recovered individuals may only have partial immunity. Then, some individuals in the recovered class $R_{1}$ relapse back into the active TB state at rate $\delta_{1}$. Also, individuals in the $R_{1}$ class who did not relapse are not totally immune to TB infection [34] and can be infected again and move into the latent class $E_{1}$ at rate $\sigma_{3}\left(1-\delta_{1}\right) \lambda$ where $\sigma_{3}$ is the factor reducing the risk of infection as a result of acquiring immunity for recovered individuals. Note that $\sigma_{3} \in(0,1)$ since primary infection confers some degree of immunity

Non-diabetics individuals acquire DM at rate $\alpha$ when the pancreas does not produce enough insulin or when the body cannot effectively use the insulin it produces. In other words, individuals in the classes without DM (those in the classes $S_{1}, E_{1}$ and $R_{1}$ ) may move to the classes with DM (those in the classes $S_{2}, E_{2}$ and $R_{2}$ ) at rate $\alpha$. Non-diabetics infectious acquire DM at rate $\tau \alpha$ where $\tau$ is a modification parameter which increases the progression of non-diabetics infectious to diabetic infectious because of their active TB status $[21,22]$.

Diabetics susceptible individuals are infected with MTB at rate $\theta \lambda$ with $\theta>1$, since diabetes act as a carrier of MTB [17]. A proportion $p_{2}$ of newly diabetic infected individuals develops a fast TB and the remainder $\left(1-p_{2}\right)$ develops a latent TB and move to the $E_{2}$ class. Let $r_{2} E_{2}$ be the number of diabetics latently infected individuals who have received a chemoprophylaxis where $r_{2}$ is the rate of chemoprophylaxis. Thus, diabetic latently infected individuals who have not received effective chemoprophylaxis progress to an active TB at rate $k_{2}\left(1-r_{2}\right)$ for endogenous reactivation and $\sigma_{2} \theta\left(1-r_{2}\right) \lambda$ for exogenous re-infection, respectively. It is worth noting here that $k_{2}>k_{1}$, since diabetic latently infected individuals tend to develop an active TB at a faster rate than non-diabetics latently infected individuals [24]. Diabetics infectious have a reduced rate of recovery if they are put on treatment and move to the $R_{2}$ class at rate $\gamma_{2}$ with $\gamma_{2} \leq \gamma_{1}$. Diabetics recovered individuals can only have partial immunity, and hence, they can undergo a reactivation of the disease at rate $\delta_{2}$ or be reinfected against with the same transmission rate $\beta$ at rate $\sigma_{4} \theta\left(1-\delta_{2}\right) \lambda$ and move into the latent class $E_{2}$, since primary infection confers some immunity where $\sigma_{4} \in(0,1)$ is the factor reducing the risk of infection as a result of acquiring immunity for diabetics recovered individuals.

The natural death rate in each class is assumed to be $\mu>0$, and infectious have additional TB induced death rates, $d_{1}>0$ and $d_{2}>0$ for active TB patients who are non-diabetics and diabetics, respectively. It is important to note here that $d_{2} \geq d_{1}$ as DM experience greater disease induced deaths than their corresponding non-diabetic counterparts [16].

The structure of the model is shown in Fig. 1. 


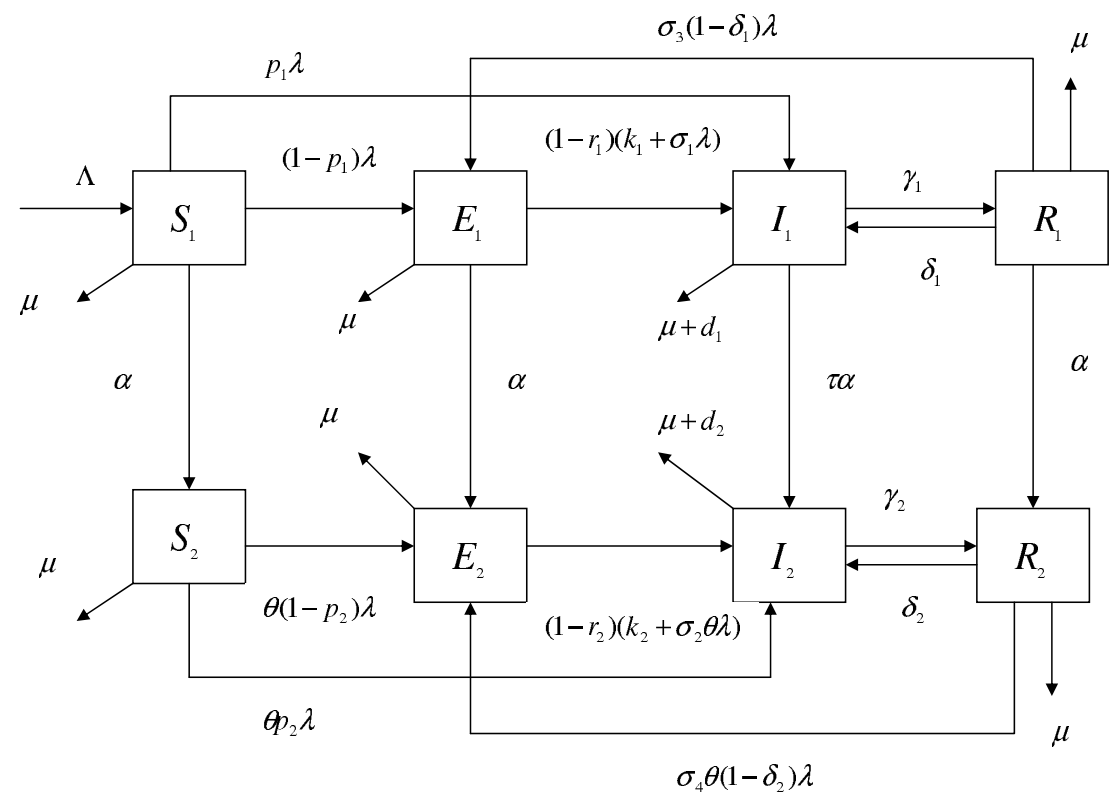

FiguRE 1. Structure of the model.

The dynamics of the disease, in a well-mixed population, is then described by the following system of nonlinear differential equations:

$$
\left\{\begin{array}{l}
\dot{S}_{1}=\Lambda-(\mu+\alpha+\lambda) S_{1}, \\
\dot{E}_{1}=\left(1-p_{1}\right) \lambda S_{1}+\sigma_{3}\left(1-\delta_{1}\right) \lambda R_{1}-\left(1-r_{1}\right)\left(k_{1}+\sigma_{1} \lambda\right) E_{1}-(\mu+\alpha) E_{1}, \\
\dot{I}_{1}=p_{1} \lambda S_{1}+\left(1-r_{1}\right)\left(k_{1}+\sigma_{1} \lambda\right) E_{1}+\delta_{1} R_{1}-\left(\mu+d_{1}+\tau \alpha+\gamma_{1}\right) I_{1}, \\
\dot{R}_{1}=\gamma_{1} I_{1}-\sigma_{3}\left(1-\delta_{1}\right) \lambda R_{1}-\left(\mu+\delta_{1}+\alpha\right) R_{1}, \\
\dot{S}_{2}=\alpha S_{1}-(\mu+\theta \lambda) S_{2}, \\
\dot{E}_{2}=\theta\left(1-p_{2}\right) \lambda S_{2}+\sigma_{4} \theta\left(1-\delta_{2}\right) \lambda R_{2}+\alpha E_{1}-\left(1-r_{2}\right)\left(k_{2}+\sigma_{2} \theta \lambda\right) E_{2}-\mu E_{2}, \\
\dot{I}_{2}=\theta p_{2} \lambda S_{2}+\left(1-r_{2}\right)\left(k_{2}+\sigma_{2} \theta \lambda\right) E_{2}+\tau \alpha I_{1}+\delta_{2} R_{2}-\left(\mu+d_{2}+\gamma_{2}\right) I_{2}, \\
\dot{R}_{2}=\gamma_{2} I_{2}-\sigma_{4} \theta\left(1-\delta_{2}\right) \lambda R_{2}+\alpha R_{1}-\left(\mu+\delta_{2}\right) R_{2} .
\end{array}\right.
$$

System (2.3) can be written in the following compact form:

$$
\left\{\begin{array}{l}
x^{\prime}(t)=\Gamma+A_{x} x(t)-\lambda \sum_{i=1}^{2} B_{i}\left\langle e_{i} \mid x(t)\right\rangle \\
y^{\prime}(t)=\lambda\left[\sum_{i=1}^{2} \mathcal{K}_{i}\left\langle e_{i} \mid x(t)\right\rangle+\sum_{i=3}^{6} B_{i}\left\langle e_{i} \mid y(t)\right\rangle\right]+A_{y} y(t),
\end{array}\right.
$$

where $x(t)=\left(x_{1}(t), x_{2}(t)\right)^{T}=\left(S_{1}(t), S_{2}(t)\right)^{T} \in \mathbb{R}_{+}^{2}, y(t)=\left(y_{1}(t), y_{2}(t), y_{3}(t), y_{4}(t), y_{5}(t), y_{6}(t)\right)^{T}=$ $\left(E_{1}(t), I_{1}(t), R_{1}(t), E_{2}(t), I_{2}(t), R_{2}(t)\right)^{T} \in \mathbb{R}_{+}^{6}, \Gamma=(\Lambda, 0)^{T}, \lambda=\frac{\langle B \mid y\rangle}{N}, B=(0, \beta, 0,0, \beta \varepsilon, 0), B_{1}=$ 
$(1,0)^{T}, B_{2}=(0, \theta)^{T}, B_{3}=\left(-\sigma_{1}\left(1-r_{1}\right), \sigma_{1}\left(1-r_{1}\right), 0,0,0,0\right)^{T}, B_{4}=\left(0,0,0,-\theta \sigma_{2}\left(1-r_{2}\right), \theta \sigma_{2}(1-\right.$ $\left.\left.r_{2}\right), 0\right)^{T}, B_{5}=\left(\sigma_{3}\left(1-\delta_{1}\right), 0,-\sigma_{3}\left(1-\delta_{1}\right), 0,0,0\right)^{T}, B_{6}=\left(0,0,0, \theta \sigma_{4}\left(1-\delta_{2}\right), 0,-\theta \sigma_{4}\left(1-\delta_{2}\right)\right)^{T}, e_{1}=(1,0)$, $e_{2}=(0,1), e_{3}=(1,0,0,0,0,0), e_{4}=(0,0,0,1,0,0), e_{5}=(0,0,1,0,0,0), e_{6}=(0,0,0,0,0,1), \mathcal{K}_{1}=$ $\left(1-p_{1}, p_{1}, 0,0,0,0\right)^{T}, \mathcal{K}_{2}=\left(0,0,0, \theta\left(1-p_{2}\right), \theta p_{2}, 0\right)^{T}$,

$$
A_{x}=\left[\begin{array}{cc}
-(\mu+\alpha) & 0 \\
\alpha & -\mu
\end{array}\right], \quad A_{y}=\left[\begin{array}{cccccc}
A_{1} & 0 & 0 & 0 & 0 & 0 \\
-k_{1}\left(1-r_{1}\right) & A_{2} & -\delta_{1} & 0 & 0 & 0 \\
0 & -\gamma_{1} & A_{3} & 0 & 0 & 0 \\
-\alpha & 0 & 0 & A_{4} & 0 & 0 \\
0 & -\tau \alpha & 0 & -k_{2}\left(1-r_{2}\right) & A_{5} & -\delta_{2} \\
0 & 0 & -\alpha & 0 & -\gamma_{2} & A_{6}
\end{array}\right],
$$

with

$$
\begin{aligned}
& A_{1}=k_{1}\left(1-r_{1}\right)+\mu+\alpha, \quad A_{2}=\mu+d_{1}+\gamma_{1}+\tau \alpha, \quad A_{3}=\mu+\delta_{1}+\alpha, \\
& A_{4}=k_{2}\left(1-r_{2}\right)+\mu, \quad A_{5}=\mu+\gamma_{2}+d_{2} \quad \text { and } \quad A_{6}=\mu+\delta_{2} .
\end{aligned}
$$

In Eq. (2.4), $\langle a \mid b\rangle=a^{T} b$ is the usual inner scalar product. The parameter values used for numerical

\begin{tabular}{|c|c|c|c|}
\hline Definition & Symbols & Estimated & Source \\
\hline Recruitment rate & $\Lambda$ & $667685 / \mathrm{yr}$ & 35 \\
\hline TB transmission coefficient & $\beta$ & {$[3,13.5]$} & {$[51]$} \\
\hline Natural mortality rate & $\mu$ & $1 / 53.5 / \mathrm{yr}$ & {$[35]$} \\
\hline Endogenous reactivation rate & & & \\
\hline of non-diabetics latent individuals & $k_{1}$ & $0.00013 / \mathrm{yr}$ & {$[38]$} \\
\hline Endogenous reactivation rate & & & \\
\hline of diabetics latent individuals & $k_{2}$ & $2 \times k_{1} / \mathrm{yr}$ & Estimated \\
\hline Chemoprophylaxis rate of non-diabetics latent individuals & $r_{1}$ & $0 / \mathrm{yr}$ & {$[36]$} \\
\hline Chemoprophylaxis rate of diabetics latent individuals & $r_{2}$ & $0 / \mathrm{yr}$ & {$[36]$} \\
\hline Recovery rate of non-diabetics infectious & $\gamma_{1}$ & $0.7372 / \mathrm{yr}$ & {$[36]$} \\
\hline Recovery rate of diabetics infectious & $\gamma_{2}$ & $0.7372 / \mathrm{yr}$ & Estimated \\
\hline Protective factor for non-diabetics latent individuals & $\sigma_{1}$ & $0.75 * p_{1}$ & {$[37]$} \\
\hline Protective factor for diabetics latent individuals & $\sigma_{2}$ & $0.7 * p_{2}$ & Assumed \\
\hline Protective factor for non-diabetics recovered individuals & $\sigma_{3}$ & $0.73 * p_{1}$ & Assumed \\
\hline Protective factor for diabetics recovered individuals & $\sigma_{4}$ & $0.71 * p_{2}$ & Assumed \\
\hline Enhancement factor & $\theta$ & 2 & {$[48]$} \\
\hline Enhancement factor & $\varepsilon$ & 1.1 & Assumed \\
\hline Enhancement factor & $\tau$ & 1.01 & Assumed \\
\hline Rate of acquiring DM & $\alpha$ & $9 / 1000 / \mathrm{yr}$ & Assumed \\
\hline TB induced mortality of non-diabetics infectious & $d_{1}$ & $0.275 / \mathrm{yr}$ & {$[36]$} \\
\hline TB induced mortality of diabetics infectious & $d_{2}$ & $1.25 * d_{1} / \mathrm{yr}$ & Estimated \\
\hline $\begin{array}{l}\text { Rate of developing fast active } \\
\text { TB among non-diabetics susceptible } S_{1} \\
\text { Rate of developing fast active TB }\end{array}$ & $p_{1}$ & 0.03 & Estimated \\
\hline among diabetics susceptible $S_{2}$ & $p_{2}$ & 0.06 & Estimated \\
\hline Relapse of non-diabetics recovered individuals & $\delta_{1}$ & $0.0986 / \mathrm{yr}$ & {$[36]$} \\
\hline Relapse of diabetics recovered individuals & $\delta_{2}$ & $0.1 / \mathrm{yr}$ & Assumed \\
\hline
\end{tabular}
simulation are given in Table 1.

Table 1: Numerical values for the parameters of model system (2.3)

\subsection{Basic properties}

\subsubsection{Positivity and boundedness of solutions}

For model system (2.3) to be epidemiologically meaningful, it is important to prove that all its state variables are non-negative for all time. In other words, solutions of model system (2.3) with positive initial data remain positive for all time $t>0$. We have the following result. 
Theorem 2.1. Let the initial data be $S_{1}(0)>0, E_{1}(0)>0, I_{1}(0)>0, R_{1}(0)>0, S_{2}(0)>0, E_{2}(0)>0$, $I_{2}(0)>0$ and $R_{2}(0)>0$. Then, the solutions $\left(S_{1}, E_{1}, I_{1}, R_{1}, S_{2}, E_{2}, I_{2}, R_{2}\right)$ of model system (2.3) are positive for all $t>0$. Furthermore,

$$
\lim _{t \rightarrow \infty} \sup N(t) \leq \frac{\Lambda}{\mu}
$$

Proof. It follows from the first equation of model system (2.3), that

$$
\frac{d S_{1}}{d t}=\Lambda-(\mu+\alpha+\lambda) S_{1}
$$

The above equation can be rewritten as,

$$
\frac{d}{d t}\left[S_{1}(t) \exp \left\{(\mu+\alpha) t+\int_{0}^{t} \lambda(s) d s\right\}\right] \geq \Lambda \exp \left\{(\mu+\alpha) t+\int_{0}^{t} \lambda(s) d s\right\} .
$$

Hence,

$$
S_{1}(\bar{t}) \exp \left\{(\mu+\alpha) \bar{t}+\int_{0}^{\bar{t}} \lambda(s) d s\right\}-S_{1}(0) \geq \int_{0}^{\bar{t}} \Lambda \exp \left\{(\mu+\alpha) u+\int_{0}^{u} \lambda(w) d w\right\} d u
$$

so that

$$
\begin{aligned}
S_{1}(\bar{t}) & \geq S_{1}(0) \exp \left\{-\left((\mu+\alpha) \bar{t}+\int_{0}^{\bar{t}} \lambda(s) d s\right)\right\} \\
& +\exp \left\{-\left((\mu+\alpha) \bar{t}+\int_{0}^{\bar{t}} \lambda(s) d s\right)\right\} \times \int_{0}^{\bar{t}} \Lambda \exp \left\{(\mu+\alpha) u+\int_{0}^{u} \lambda(w) d w\right\} d u>0 .
\end{aligned}
$$

Similarly, it can be shown that $E_{1}(t)>0, I_{1}(t)>0, R_{1}(t)>0, S_{2}(t)>0, E_{2}(t)>0, I_{2}(t)>0$ and $R_{2}(t)>0$ for all $t>0$.

On the other hand, adding all the equations in the differential system (2.3) gives

$$
N^{\prime}(t)=\Lambda-\mu N-d_{1} I_{1}-d_{2} I_{2} .
$$

It follows from Eq. (2.6) that

$$
\Lambda-\left(\mu+d_{1}+d_{2}\right) N \leq N^{\prime}(t) \leq \Lambda-\mu N .
$$

Thus,

so that

$$
\frac{\Lambda}{\mu+d_{1}+d_{2}} \leq \lim _{t \rightarrow \infty} \inf N(t) \leq \lim _{t \rightarrow \infty} \sup N(t) \leq \frac{\Lambda}{\mu}
$$

$$
\lim _{t \rightarrow \infty} \sup N(t) \leq \frac{\Lambda}{\mu} .
$$

This completes the proof.

\subsubsection{Invariant region}

Model system (2.3) will be analyzed in a suitable region as follows. We first show that model system (2.3) is dissipative. That is, all solutions are uniformly bounded in a proper subset $\Omega \subset \mathbb{R}_{+}^{8}$. Let $\left(S_{1}, E_{1}, I_{1}, R_{1}, S_{2}, E_{2}, I_{2}, R_{2}\right) \in \mathbb{R}_{+}^{8}$ be any solution with non-negative initial conditions.

Model system $(2.3)$ has a varying population size $(N \neq 0)$ and therefore a trivial equilibrium is not feasible. Let $\rho=\min \left(d_{1}, d_{2}\right)$, then, from model system (2.3), it follows that

$$
\begin{aligned}
N^{\prime}(t) & =\Lambda-\mu N-d_{1} I_{1}-d_{2} I_{2}, \\
& \leq \Lambda-\mu N(t)-\rho\left(I_{1}(t)+I_{2}(t)\right), \\
& \leq \Lambda-\mu N(t) .
\end{aligned}
$$


So that (cf. [40])

$$
0 \leq N(t) \leq \frac{\Lambda}{\mu}+\left(N(0)-\frac{\Lambda}{\mu}\right) e^{-\mu t},
$$

where $N(0)$ represents the value of $N(t)$ evaluated at the initial values of the respective variables. The lower limit comes naturally from the fact that the model variables and parameters are non-negative $(t \geq 0)$ since they monitor human populations. Thus, as $t \rightarrow \infty, 0 \leq N(t) \leq \Lambda / \mu$. Therefore, all feasible solutions of model system (2.3) enter the region:

$$
\Omega=\left\{\left(S_{1}, E_{1}, I_{1}, R_{1}, S_{2}, E_{2}, I_{2}, R_{2}\right) \in \mathbb{R}_{+}^{8}, N(t) \leq \frac{\Lambda}{\mu}\right\} .
$$

Thus, model system (2.3) is mathematically and epidemiologically well-posed and it is sufficient to consider the dynamics of the flow generated by model system (2.3) in $\Omega$.

We have the following result.

Theorem 2.2. For every non-zero, non-negative initial value, solutions of model system (2.3) exist for all times.

Proof. Local existence of solutions follows from standard arguments since the right-hand side of model system (2.3) is locally Lipschitz continuous. Global existence follows from a priori bounds. This concludes the proof.

\section{Mathematical analysis}

\subsection{Stability of the disease-free equilibrium (DFE)}

\subsubsection{The basic reproduction number and its analysis}

Model system (2.3) has a disease-free equilibrium, obtained by setting the right-hand sides of equations in the model to zero, given by

$$
Q_{0}=\left(S_{1}^{0}, 0,0,0, S_{2}^{0}, 0,0,0\right)
$$

where

$$
S_{1}^{0}=\frac{\Lambda}{\mu+\alpha} \quad \text { and } \quad S_{2}^{0}=\frac{\Lambda}{\mu} \frac{\alpha}{\mu+\alpha} .
$$

The linear stability of $Q_{0}$ can be established using the next generation operator method on model system (2.3). Using the same notation as in [41], the matrices $F$ and $V$, for the new infection terms and the remaining transfer terms, are, respectively, given by

$$
F=\frac{1}{N_{0}} \sum_{i=1}^{2} \mathcal{K}_{i} B S_{i}^{0} \quad \text { and } \quad V=-A_{y},
$$

where $N_{0}=\Lambda / \mu$, giving

$$
\mathcal{R}_{0}=\rho\left(F V^{-1}\right)=\frac{1}{N_{0}} \sum_{i=1}^{2}\left\langle B \mid\left(-A_{y}\right)^{-1} \mathcal{K}_{i}\right\rangle S_{i}^{0},
$$

where $\rho$ represents the spectral radius.

The result below (Lemma 3.1) follows from Theorem 2 of [43].

Lemma 3.1. The disease-free equilibrium $Q_{0}$ of model system (2.3) is locally asymptotically stable whenever $\mathcal{R}_{0}<1$, and unstable whenever $\mathcal{R}_{0}>1$. 
The threshold quantity $\mathcal{R}_{0}$ is the basic reproduction number for TB infection in the presence of DM. It measures the average number of new TB infections generated by a single infectious in a completely susceptible population. Consequently, the disease-free equilibrium of model system (2.3) is locally asymptotically stable (LAS) whenever $\mathcal{R}_{0}<1$ and unstable if $\mathcal{R}_{0}>1$. This means that TB can be eliminated from the community (when $\mathcal{R}_{0}<1$ ) if the sizes of the population of model system $(2.3)$ are in the basin of attraction of the disease-free equilibrium $Q_{0}$.

Now, suppose that there are non diabetics in the community. In this case $\theta=\alpha=0$ and $S_{2}=E_{2}=$ $I_{2}=R_{2}=0$. Following van den Driessche and Watmough [43], the basic reproduction number is given by

$$
\mathcal{R}_{0}^{T}=\frac{\beta\left(\mu+\delta_{1}\right)\left[k_{1}\left(1-r_{1}\right)+\mu p_{1}\right]}{\left[k_{1}\left(1-r_{1}\right)+\mu\right]\left[\mu\left(\mu+d_{1}+\gamma_{1}\right)+\delta_{1}\left(\mu+d_{1}\right)\right]} .
$$

From the above expression, on can easily see that when the treatment rate $\gamma_{1}$ increasing, the basic reproduction number $\mathcal{R}_{0}^{T}$ decreases. This means that the treatment of active TB patients in a nondiabetics population would have a positive impact in TB control.

Now, let us analyze the basic reproduction number. The following numerical results demonstrate the role of $\theta, \tau$ and $\alpha$ on the basic reproduction number $\mathcal{R}_{0}$.

We begin by investigating how the basic reproduction number $\mathcal{R}_{0}$ depends on $\alpha$ and $\theta$ when $\beta=5$ using parameter values in Table 1. The illustration in Fig. 2 shows that the increased of the rate to become diabetic $\alpha$ and the susceptibility of TB du to DM, $\theta$ results in an increase in $\mathcal{R}_{0}$. This figure also illustrate that for the chosen parameter values, if the susceptibility to TB due to DM $\theta$ does not exceed $2.7(\theta<1.3)$, then TB can be controlled irrespective of the value of $\alpha$. The infection will equally persist for $\theta>1.3$.

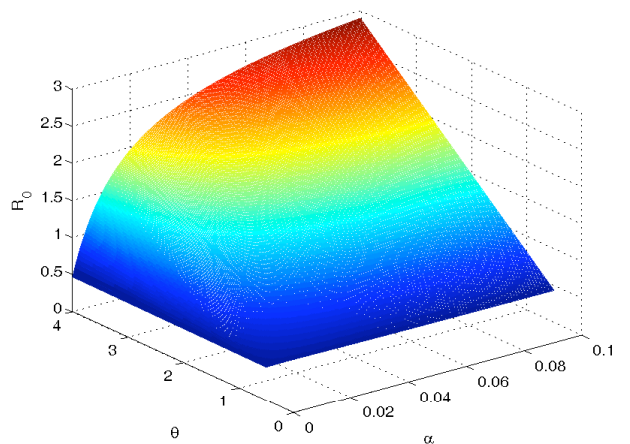

(a)

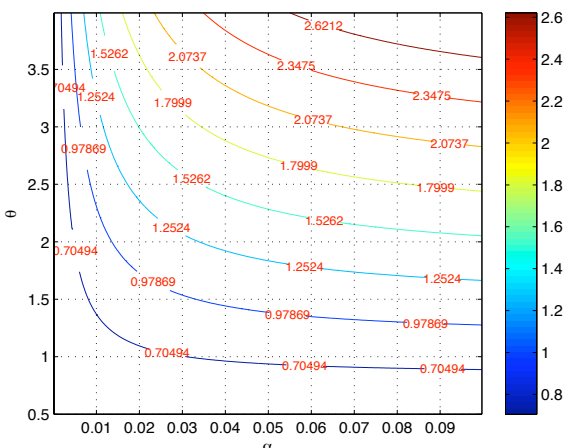

(b)

Figure 2. (a) 3-D and (b) contour plot showing effects of $\theta$ and $\alpha$ on the basic reproduction ratio $\mathcal{R}_{0}$ when $\beta=5$. All other parameters are as in Table 1.

We now investigate how the basic reproduction number $\mathcal{R}_{0}$ depends on the modification parameter $\tau$ and the susceptibility to TB due to DM when $\beta=5$ (see Fig. 3). This figure is reflecting a large state number of TB cases. The high $\theta$ and low $\tau$ may increase the spread of TB, while low $\theta$ and high $\tau$ may reduce the spread of $\mathrm{TB}$ in the community.

\subsubsection{Sensitivity analysis}

Sensitivity analysis is used to determine the relative importance of model parameters to TB transmission and its prevalence. We perform the analysis by calculating the sensitivity indices of the basic reproduction number, $\mathcal{R}_{0}$. According to [47], sensitivity analysis is commonly used to determine the robustness of model predictions to parameter values, since there are usually errors in data collection and 


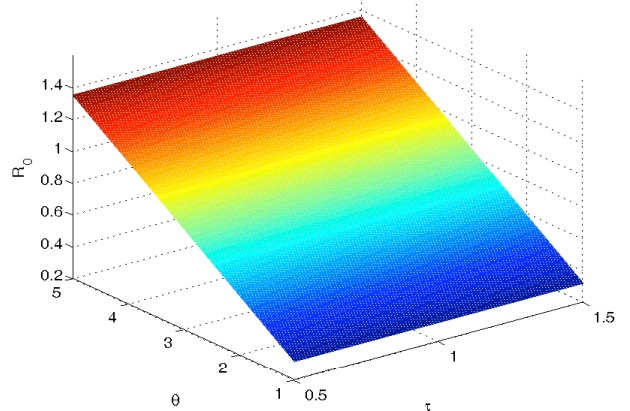

(a)

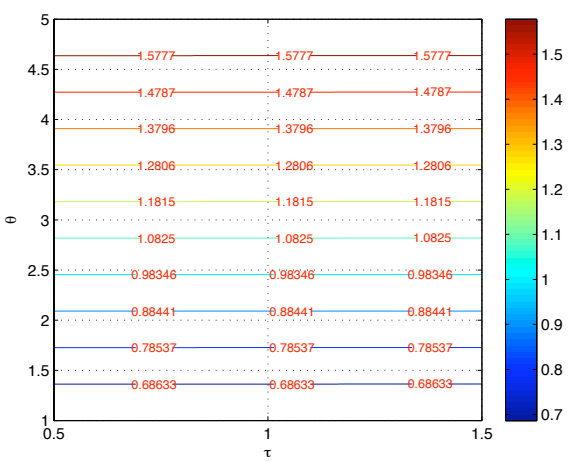

(b)

FiguRE 3. (a) 3-D and (b) contour plot showing effects of $\theta$ and $\tau$ on the basic reproduction ratio $\mathcal{R}_{0}$ when $\beta=5$. All other parameters are as in Table 1 .

estimated values. We are thus interested in parameters that significantly affect the model reproduction number since these are the parameters that should be taken into consideration when considering intervention strategies. Sensitivity analysis also permits us to measure the relative change in a state variable when a parameter changes. The normalized forward sensitivity index of a variable to a parameter is the ratio of the relative change in the variable to the relative change in the parameter. Since the reproduction number is a differentiable function of the parameters, the sensitivity index may alternatively be defined using partial derivatives. For instance, the computation of the sensitivity index of $\mathcal{R}_{0}$ with respect to $\beta$ using the parameter values in the table 2 is given by

$$
\prod_{\beta}^{\mathcal{R}_{0}}=\left(\frac{\partial \mathcal{R}_{0}}{\partial \beta}\right)\left(\frac{\beta}{\mathcal{R}_{0}}\right)=1>0 .
$$

This shows that $\mathcal{R}_{0}$ is an increasing function of $\beta$ and the parameter $\beta$ has a strong influence on the spread of TB. We tabulate the indices of the remaining parameters in Table 2.

Table 2: Sensitivity indices for the basic reproduction number $\mathcal{R}_{0}$

\begin{tabular}{llllll}
\hline Parameter & Index & Parameter & Index & Parameter & Index \\
\hline$\beta$ & 1 & $\theta$ & 0.7780 & $\Lambda$ & 0 \\
$\varepsilon$ & 0.8806 & $p_{1}$ & 0.2089 & $p_{2}$ & 0.7320 \\
$k_{1}$ & 0.0033 & $k_{2}$ & 0.0047 & $\tau$ & 0.00032638 \\
$d_{1}$ & -0.0346 & $d_{2}$ & -0.2689 & $r_{1}$ & 0 \\
$r_{2}$ & 0 & $\delta_{1}$ & 0.0485 & $\delta_{2}$ & 0.4323 \\
$\gamma_{1}$ & -0.0731 & $\gamma_{2}$ & -0.5032 & $\mu$ & -0.5935 \\
$\alpha$ & 0.1794 & $\sigma_{1}$ & 0 & $\sigma_{2}$ & 0 \\
$\sigma_{3}$ & 0 & $\sigma_{4}$ & 0 & & \\
\hline
\end{tabular}

From Table 2, parameters whose sensitivity indices have negative signs decrease the value of the basic reproduction number as their values increase, while those with positive signs increase the value of $\mathcal{R}_{0}$ as they increase. Those with no signs have no effect on the value of the basic reproduction number. The system is most sensitive to $\mu$, followed by $\gamma_{2}$. It is important to note that increasing (decreasing) $\mu$ by $10 \%$ decreases (increases) $\mathcal{R}_{0}$ by $5.935 \%$. However, increasing (decreasing) the parameters $\beta$ by $10 \%$ increases (decreases) $\mathcal{R}_{0}$ by $10 \%$.

\subsubsection{Stability of the disease-free equilibrium (DFE)}

Herein, we will show that the disease-free equilibrium may not be globally asymptotically stable in the case that the basic reproduction number is less than the unity $\left(\mathcal{R}_{0}<1\right)$. To this end, we will used 
the result of Castillo-Chavez et al. [45] on the global stability of the disease-free equilibrium for a class of epidemiological model. Remind that from proof in Lemma 3.1, the disease-free equilibrium $Q_{0}$ of model system (2.3) is locally asymptotically stable for $\mathcal{R}_{0}<1$ from Theorem 2 of van den Driesshes and Watmough [43].

Following Castillo-Chavez et al. [45], we rewrite model system (2.3) as

$$
\left\{\begin{array}{l}
\left.X^{\prime}(t)=F X, Y\right) \\
Y^{\prime}(t)=G(X, Y), \quad G(X, 0)=0
\end{array}\right.
$$

where $X \in \mathbb{R}_{+}^{2}$ is the vector whose components are the number of non diabetics and diabetics susceptible individuals and $Y \in \mathbb{R}_{+}^{6}$ denoting (its components) the number of non diabetics and diabetics infected individuals including latent, infectious and recovered individuals. The DFE is now denoted by $Q_{0}=$ $\left(X_{0}, 0\right)$ where $X_{0}=\left(x_{1}^{0}, x_{2}^{0}\right)$ with $x_{1}^{0}=\frac{\Lambda}{\mu+\alpha}$ and $x_{2}^{0}=\frac{\Lambda}{\mu} \frac{\alpha}{\mu+\alpha}$.

The conditions $\left(H_{1}\right)$ and $\left(H_{2}\right)$ below must be met to guarantee the global asymptotic stability of $Q_{0}$.

$$
\begin{array}{ll}
H_{1}: & \text { For } \quad X^{\prime}(t)=F(X, 0), \quad X_{0} \text { is globally asymptotically stable (GAS), } \\
H_{2}: & G(X, Y)=A Y-\hat{G}(X, Y), \quad \hat{G}(X, Y) \geq 0 \quad \text { for } \quad(X, Y) \in \Omega,
\end{array}
$$

where $A=D_{Y} G\left(X_{0}, 0\right)$ and $\Omega$ is the region where the model makes biological sense.

If model system (2.3) satisfies the conditions in Eq. (3.6), then the following result holds.

Theorem 3.2. The fixed point $Q_{0}=\left(X_{0}, 0\right)$ is a globally asymptotically stable equilibrium of model system (2.3) provided that $\mathcal{R}_{0}<1$ and the conditions in Eq. (3.6) are satisfied.

From the proof in Lemma 1 , one knows that $Q_{0}$ is locally asymptotically stable for $\mathcal{R}_{0}<1$.

Using model system (3.5), one can deduce that

$$
F(X, 0)=\left[\begin{array}{c}
\Lambda-(\mu+\alpha) S_{1} \\
\alpha S_{1}-\mu S_{2}
\end{array}\right], \quad A=\left[\begin{array}{cccc}
-A_{1} & \left(1-p_{1}\right) \beta+\gamma & \alpha & \left(1-p_{1}\right) \varepsilon \beta \\
\left(1-r_{1}\right) k_{1} & p_{1} \beta-A_{2} & 0 & p_{1} \varepsilon \beta \\
\alpha & \left(1-p_{2}\right) \theta \beta & -A_{4} & \theta\left(1-p_{2}\right) \varepsilon \beta \\
0 & p_{2} \theta \beta+\tau \alpha & \left(1-r_{2}\right) k_{2} & p_{2} \theta \varepsilon \beta-A_{5}
\end{array}\right],
$$

where $A_{1}, A_{2}, A_{3}$ and $A_{4}$ are defined as in Eq. (2.4) and

$$
\widehat{G}(x, y)=\left(\begin{array}{c}
\left(1-p_{1}\right) N \lambda\left(1-\frac{S_{1}}{N}\right)+\sigma_{1}\left(1-r_{1}\right) \lambda E_{1}-\sigma_{3}\left(1-\delta_{1}\right) \lambda R_{1} \\
p_{1} N \lambda\left(1-\frac{S_{1}}{N}\right)-\sigma_{1}\left(1-r_{1}\right) \lambda E_{1} \\
\theta\left(1-p_{2}\right) N \lambda\left(1-\frac{S_{2}}{N}\right)+\theta \sigma_{2}\left(1-r_{2}\right) \lambda E_{2}-\sigma_{4}\left(1-\delta_{2}\right) \lambda R_{2} \\
\theta p_{2} N \lambda\left(1-\frac{S_{2}}{N}\right)-\theta \sigma_{2}\left(1-r_{2}\right) \lambda E_{2}
\end{array}\right)=\left(\begin{array}{l}
\widehat{G}_{1}(x, y) \\
\widehat{G}_{2}(x, y) \\
\widehat{G}_{3}(x, y) \\
\widehat{G}_{4}(x, y)
\end{array}\right) .
$$

Note that $A$ is an M-matrix (the off diagonal elements of $A$ are nonnegative) [41,42]. Thus, if $Y(0) \in \Omega$, then $Y(t) \in \Omega$. Indeed, there exist $s>0$ and $C \geq 0$ such that

$$
-A=s I-C,
$$

which implies that $-A$ is a singular irreducible M-matrix. Thus, following Theorem 4.16, page 156 in [41], there exists a positive vector $\tilde{Y}>>0$ such that

$$
A \tilde{Y}=0 .
$$


From Perron-Froebenius theorem, we deduce that there exist a simple eigenvalue $\lambda_{\max }=\max _{\lambda \in \operatorname{sp}(A)} \mathcal{R}_{e}(\lambda)$ and an eigenvector $\omega>>0$ such that

$$
A \omega=\lambda_{\max } \omega .
$$

Now, using the fact that $\mathbf{1}^{T} A=0$, where $\mathbf{1}^{T}=(1,1,1,1,1,1)$, we have $\lambda_{\max }=0$, and thus all other eigenvalues have a negative real part. Thus, one can deduce that the linearized system $\dot{Y}=A Y$ admits a unique positive equilibrium 0 which is locally asymptotically stable on the hyperplane orthogonal to $\mathbf{1}^{T}$. Replacing the vector 0 in the first equation of system (3.5) gives $X^{\prime}(t)=F(X, 0)$. Then, one can prove that $X_{0}$ is globally asymptotically stable on $\Omega$ and condition $H_{1}$ is satisfied.

The sign of $\widehat{G}_{i}(x, y), \mathrm{i}=1,2,3$ or 4 is not obvious, but based on the model parameters, $G(x, y)$ is neither positive nor equal to zero. Condition $\left(\mathrm{H}_{2}\right)$ in $(3.6)$ is therefore violated and as such, $Q_{0}$ may not be a globally asymptotically stable. But, in the absence of exogenous re-infection and endogenous reactivation and relapse of recovered individuals (i.e., $\sigma_{1}=\sigma_{2}=\sigma_{3}=\sigma_{4}=\delta_{1}=\delta_{2}=0$ ) $Q_{0}$ is globally asymptotically stable.

\subsection{Endemic equilibria and stability analysis}

Model system (2.3) has basically two possible endemic equilibria, that is non diabetic only endemic equilibrium when there are non-diabetics in the community and the equilibrium point where both nondiabetics and diabetics co-exist, herein referred to as the interior equilibrium point. It is worth mentioning that the endemic equilibrium where diabetics only exist is an unrealistic equilibrium point and for that reason it is not discussed in this paper.

\subsubsection{Non-diabetics TB only equilibrium point}

This equilibrium in terms of the force of infection $\lambda^{*}$ is given by

$$
Q^{*}=\left(S_{1}^{*}, E_{1}^{*}, I_{1}^{*}, R_{1}^{*}, 0,0,0,0\right),
$$

where

$$
\begin{aligned}
& S_{1}^{*}=\frac{\Lambda}{\mu+\lambda^{*}}, \quad I_{1}^{*}=\frac{\Lambda \lambda^{*}}{\beta \mu+d_{1} \lambda^{*}}, \quad R_{1}^{*}=\frac{\gamma_{1} \Lambda \lambda^{*}}{\left[\sigma_{3}\left(1-\delta_{1}\right) \lambda^{*}+\mu+\delta_{1}\right]\left(\beta \mu+d_{1} \lambda^{*}\right)}, \\
& E_{1}^{*}=\frac{\Lambda \lambda^{*}\left[\left(1-p_{1}\right)\left(\beta \mu+d_{1} \lambda^{*}\right)\left(\sigma_{3}\left(1-\delta_{1}\right) \lambda+\mu+\delta_{1}\right)+\sigma_{3}\left(1-\delta_{1}\right) \lambda^{*} \gamma_{1}\left(\mu+\lambda^{*}\right)\right]}{\left(\mu+\lambda^{*}\right)\left(\beta \mu+d_{1} \lambda^{*}\right)\left[\sigma_{3}\left(1-\delta_{1}\right) \lambda^{*}+\mu+\delta_{1}\right]\left[\mu+k_{1}\left(1-r_{1}\right)+\sigma\left(1-r_{1}\right) \lambda^{*}\right]}
\end{aligned}
$$

In Eq. (3.8), $\lambda^{*}$ satisfy the following cubic equation:

$$
c_{3}\left(\lambda^{*}\right)^{3}+c_{2}\left(\lambda^{*}\right)^{2}+c_{1} \lambda^{*}+c_{0}=0
$$

where

$$
\begin{aligned}
c_{3} & =\sigma_{3}\left(1-\delta_{1}\right) \sigma_{1} \mu\left(1-r_{1}\right) \\
c_{2} & =\sigma_{1}\left(1-r_{1}\right)\left(\gamma_{1}+\mu+\delta_{1}\right)+\sigma_{1}\left(1-r_{1}\right) \sigma_{3}\left(1-\delta_{1}\right)(-\beta+\mu+1)+\sigma_{3}\left(1-\delta_{1}\right)\left(\gamma_{1}+k_{1}\left(1-r_{1}\right)+\mu+d_{1}\left(1-p_{1}\right)\right) \\
c_{1} & =\left(k_{1}\left(1-r_{1}\right)+\mu\right)\left(\gamma_{1}+\left(\mu+\delta_{1}\right)+\sigma_{3}\left(1-\delta_{1}\right)\left(-\beta+\mu+d_{1}\right)\right)+\left(\mu+\delta_{1}\right)\left(\sigma_{1}\left(1-r_{1}\right)\left(-\beta+\mu+d_{1}\right)\right. \\
& \left.+d_{1}\left(1-p_{1}\right)\right)+\mu \gamma_{1}\left(\sigma_{1}\left(1-r_{1}\right)+\sigma_{3}\left(1-\delta_{1}\right)\right)+\beta \mu \sigma_{3}\left(1-\delta_{1}\right)\left(1-p_{1}\right) \\
c_{0} & =\left(k_{1}\left(1-r_{1}\right)+\mu\right)\left(\mu\left(\mu+d_{1}+\gamma_{1}\right)+\delta_{1}\left(\mu+d_{1}\right)\right)\left(1-\mathcal{R}_{0}^{T}\right)
\end{aligned}
$$

where $\mathcal{R}_{0}^{T}$ is defined as in Eq. (3.3). The cubic equation (3.9) can be analyzed for the possibility of multiple endemic equilibria. It is worth noting that the coefficient $c_{3}$ is always positive, and $c_{0}$ is positive (negative) if $\mathcal{R}_{0}^{T}$ is less than (greater than) the unity, respectively. 
Solving the cubic equation (3.9) yields

$$
\lambda^{*}=\frac{1}{3 c_{3}}\left[-c_{2}+\left(\frac{D_{0}}{2}+\sqrt{\left(3 c_{1} c_{3}-c_{2}\right)^{3}+D_{0}^{2}}\right)^{\frac{1}{3}}+\left(\frac{c_{0}}{2}-\sqrt{\left(3 c_{1} c_{3}-c_{2}\right)^{3}+D_{0}^{2}}\right)^{\frac{1}{3}}\right],
$$

where $D_{0}=\left(27 c_{0} c_{3}^{2}+2 c_{0}^{3}-9 c_{1} c_{2} c_{3}\right)$ and the remaining roots are complex roots which are discarded because we are dealing with a population of individuals and is always positive. We only consider conditions for $c_{2}, c_{1}$ and $c_{0}$ which gives a positive $\lambda^{*}$ since a negative force of infection is epidemiologically irrelevant. We claim the following result.

Lemma 3.3. Model system (2.3) without DM could have a unique endemic equilibrium whenever $\mathcal{R}_{0}^{T}>1$.

In order to analyze the stability of the endemic equilibrium point, we make use of the Centre Manifold theory [45] as described by Theorem 4.1 of Castillo-Chavez and Song [27] to establish the local asymptotic stability of the non-diabetics only TB endemic equilibrium. To apply this theory, the following simplification and change of variables are made first of all. Let $z_{1}=S_{1}, z_{2}=E_{1}, z_{3}=I_{1}$ and $z_{4}=R_{1}$ so that $N=z_{1}+z_{2}+z_{3}+z_{4}$. Further, by using the vector notation $z=\left(z_{1}, z_{2}, z_{3}, z_{4}\right)^{T}$, the TB model (2.3) without DM can be written in the form $\dot{z}=f(z)$, with $f=\left(f_{1}, f_{2}, f_{3}, f_{4}\right)^{T}$, as follows:

$$
\left\{\begin{array}{l}
z_{1}^{\prime}=f_{1}=\Lambda-(\mu+\lambda) z_{1}, \\
z_{2}^{\prime}=f_{2}=\left(1-p_{1}\right) \lambda z_{1}+\sigma_{3}\left(1-\delta_{1}\right) z_{4}-\left(1-r_{1}\right) \sigma_{1} \lambda z_{2}-B_{1} z_{2}, \\
z_{3}^{\prime}=f_{3}=p_{1} \lambda z_{1}+\left(1-r_{1}\right)\left(k+\sigma_{1} \lambda\right) z_{2}+\delta_{1} z_{4}-B_{2} z_{3}, \\
z_{4}^{\prime}=f_{4}=\gamma_{1} z_{3}-\sigma_{3}\left(1-\delta_{1}\right) \lambda z_{4}-B_{3} z_{4},
\end{array}\right.
$$

where

$$
\lambda=\frac{\beta z_{3}}{z_{1}+z_{2}+z_{3}+z_{4}}, \quad B_{1}=k_{1}\left(1-r_{1}\right)+\mu, \quad B_{2}=\mu+\gamma_{1}+d_{1} \text { and } B_{3}=\mu+\delta_{1} .
$$

System (3.11) has a DFE given by $Q_{0}^{1}=\left(z_{1}^{0}, 0,0,0\right)$ where $z_{1}^{0}=\Lambda / \mu$. The Jacobian of system (3.11), at the DFE $Q_{0}^{1}$, is given by

$$
J\left(Q_{0}^{1}\right)=\left(\begin{array}{cccc}
-\mu & 0 & -\beta & 0 \\
0 & -B_{1} & \beta\left(1-p_{1}\right) & 0 \\
0 & k_{1}\left(1-r_{1}\right) & \beta p_{1}-B_{2} & \delta_{1} \\
0 & 0 & \gamma_{1} & -B_{3}
\end{array}\right)
$$

The basic reproduction number of the transformed (linearized) model system (3.11) is the same as that of the original model without DM given by Eq. (3.3). Therefore, choosing $\beta$ as a bifurcation parameter by solving for $\beta$ when $\mathcal{R}_{0}^{T}=1$, we obtain

$$
\beta=\beta^{*}=\frac{\left[k_{1}\left(1-r_{1}\right)+\mu\right]\left[\mu\left(\mu+d_{1}+\gamma_{1}\right)+\delta_{1}\left(\mu+d_{1}\right)\right]}{\left(\mu+\delta_{1}\right)\left[k_{1}\left(1-r_{1}\right)+\mu p_{1}\right]} .
$$

It follows that the Jacobian $\left(J\left(Q_{0}^{1}\right)\right)$ of system $(3.11)$ at the DFE $Q_{0}^{1}$, with $\beta=\beta^{*}$, denoted by $J_{\beta^{*}}$ has a simple zero eigenvalue (with all other eigenvalues having negative real parts). Hence, the Centre Manifold theory [45] can be used to analyze the dynamics of system (3.11). In particular, the theorem in Castillo and Song [27], reproduced below for convenience, will be used to show that when $\mathcal{R}_{0}^{T}>1$, there exists a unique endemic equilibrium of system (3.11) (as shown in Lemma 3.3) which is locally asymptotically stable for $\mathcal{R}_{0}^{T}$ near 1 under certain conditions.

Theorem 3.4. (Castillo-Chavez \& Song [27]) Consider the following general system of ordinary differential equations with a parameter $\phi$ :

$$
\frac{d z}{d t}=f(x, \phi), \quad f: \mathbb{R}^{n} \times \mathbb{R} \rightarrow \mathbb{R} \quad \text { and } \quad f \in C^{2}\left(\mathbb{R}^{n}, \mathbb{R}\right),
$$

where 0 is an equilibrium point of the system (that is, $f(0, \phi) \equiv 0$ for all $\phi$ ) and assume 
1. $A=D_{z} f(0,0)=\left(\frac{\partial f_{i}}{\partial z_{j}}(0,0)\right)$ is the linearization matrix of system (3.12) around the equilibrium 0 with $\phi$ evaluated at 0 . Zero is a simple eigenvalue of $A$ and other eigenvalues of $A$ have negative real parts;

2. Matrix A has a right eigenvector $u$ and a left eigenvector $v$ (each corresponding to the zero eigenvalue).

Let $f_{k}$ be the $k^{\text {th }}$ component of $f$ and

$$
\begin{aligned}
& a=\sum_{k, i, j=1}^{n} v_{k} u_{i} u_{j} \frac{\partial^{2} f_{k}}{\partial x_{i} \partial x_{j}}(0,0), \\
& b=\sum_{k, i=1}^{n} v_{k} u_{i} \frac{\partial^{2} f_{k}}{\partial x_{i} \partial \phi}(0,0),
\end{aligned}
$$

then, the local dynamics of the system around the equilibrium point 0 is totally determined by the signs of $a$ and $b$.

1. $a>0, b>0$. When $\phi<0$ with $|\phi| \ll 1,0$ is locally asymptotically stable and there exists a positive unstable equilibrium; when $0<\phi \ll 0,0$ is unstable and there exists a negative, locally asymptotically stable equilibrium;

2. $a<0, b<0$. When $\phi<0$ with $|\phi| \ll 1,0$ is unstable; when $0<\phi \ll 1,0$ is locally asymptotically stable equilibrium, and there exists a positive unstable equilibrium;

3. $a>0, b<0$. When $\phi<0$ with $|\phi| \ll 1,0$ is unstable, and there exists a locally asymptotically stable negative equilibrium; when $0<\phi \ll 1,0$ is stable, and a positive unstable equilibrium appears;

4. $a<0, b>0$. When $\phi$ changes from negative to positive, 0 changes its stability from stable to unstable. Correspondingly a negative unstable equilibrium becomes positive and locally asymptotically stable.

Particularly, if $a>0$ and $b>0$, then a backward bifurcation occurs at $\phi=0$.

In order to apply the above theorem, the following computations are necessary (it should be noted that we are used $\beta^{*}$ as the bifurcation parameter, in place of $\phi$ in Theorem [27]).

Eigenvectors of $J_{\beta^{*}}$ : For the case when $\mathcal{R}_{0}^{T}=1$, it can be shown that the Jacobian of system (3.11) at $\beta=\beta^{*}$ (denoted by $J_{\beta^{*}}$ ) has a right eigenvector (corresponding to the zero eigenvalue), given by $U=\left(u_{1}, u_{2}, u_{3}, u_{4}\right)^{T}$, where,

$$
u_{1}=-\frac{\beta}{\mu} u_{3}, \quad u_{2}=\frac{\beta\left(1-p_{1}\right)}{B_{1}} u_{3}, \quad u_{3}=u_{3}>0 \quad \text { and } \quad u_{4}=\frac{\gamma_{1}}{B_{3}} u_{3} .
$$

Similarly, the components of the left eigenvectors of $J_{\beta^{*}}$ (corresponding to the zero eigenvalue), denoted by $V=\left(v_{1}, v_{2}, v_{3}, v_{4}\right)^{T}$, are given by,

$$
v_{1}=0, \quad v_{2}=\frac{k_{1}\left(1-r_{1}\right) v_{3}}{B_{1}}, \quad v_{3}=v_{3}>0 \quad \text { and } \quad v_{4}=\frac{\delta_{1}}{B_{3}} v_{3} .
$$

Computation of $\mathbf{b}$ : For the sign of $b$, it can be shown that the associated non-vanishing partial derivatives of $f$ are

$$
\frac{\partial^{2} f_{1}}{\partial z_{3} \partial \beta^{*}}=-1, \quad \frac{\partial^{2} f_{2}}{\partial z_{3} \partial \beta^{*}}=1-p_{1} \quad \text { and } \quad \frac{\partial^{2} f_{3}}{\partial z_{3} \partial \beta^{*}}=p_{1} .
$$

Substituting the respective partial derivatives into the expression

$$
b=v_{2} \sum_{i=1}^{4} u_{i} \frac{\partial^{2} f_{2}}{\partial z_{i} \beta^{*}}+v_{3} \sum_{i=1}^{3} u_{i} \frac{\partial^{2} f_{3}}{\partial z_{i} \beta^{*}},
$$

gives

$$
b=\frac{\left[p_{1} \mu+k_{1}\left(1-r_{1}\right)\right] u_{3} v_{3}}{B_{1}}>0 .
$$


Computation of $a$ : For system (3.11), the associated non-zero partial derivatives of $f$ (at the DFE $Q_{0}^{1}$ ) are given by

$$
\begin{array}{ll}
\frac{\partial^{2} f_{2}}{\partial z_{3} \partial z_{2}}=-\frac{\beta^{*} \mu\left(\sigma_{1}\left(1-r_{1}\right)+\left(1-p_{1}\right)\right)}{\Lambda}, & \frac{\partial^{2} f_{2}}{\partial z_{3}^{2}}=-\frac{\beta^{*}\left(1-p_{1}\right) \mu}{\Lambda}, \\
\frac{\partial^{2} f_{2}}{\partial z_{3} \partial z_{4}}=\frac{\beta^{*} \mu\left(\sigma_{3}\left(1-\delta_{1}\right)-\left(1-p_{1}\right)\right)}{\Lambda}, & \frac{\partial^{2} f_{3}}{\partial z_{3} \partial z_{2}}=\frac{\beta^{*} \mu\left(\sigma_{1}\left(1-r_{1}\right)-p_{1}\right)}{\Lambda}, \\
\frac{\partial^{2} f_{3}}{\partial z_{3}^{2}}=-\frac{\beta^{*} p_{1} \mu}{\Lambda}, \quad \frac{\partial^{2} f_{3}}{\partial z_{4} \partial z_{3}}=-\frac{\beta^{*} p_{1} \mu}{\Lambda} & \text { and } \quad \frac{\partial^{2} f_{4}}{\partial z_{4} \partial z_{3}}=-\frac{\beta^{*} \mu \sigma_{3}\left(1-\delta_{1}\right)}{\Lambda} .
\end{array}
$$

Then, it follows that

$$
\begin{aligned}
a & =v_{2} \sum_{i, j=1}^{4} u_{i} u_{j} \frac{\partial^{2} f_{2}}{\partial z_{i} \partial z_{j}}+v_{4} \sum_{i, j=1}^{3} u_{i} u_{j} \frac{\partial^{2} f_{3}}{\partial z_{i} \partial z_{j}}+v_{4} \sum_{i, j=1}^{3} u_{i} u_{j} \frac{\partial^{2} f_{4}}{\partial z_{i} \partial z_{j}} \\
& =-\frac{\beta^{*} u_{3}^{2} v_{3} \mu}{B_{1}^{2} B_{3}}\left[p_{1} \mu+k_{1}\left(1-r_{1}\right)\right]\left[B_{3}\left(\beta^{*}\left(1-p_{1}\right)+B_{1}\right)+2 \gamma_{1} B_{1}\right] \\
& +2 \frac{\beta^{*} u_{3}^{2} v_{3} \mu}{B_{1}^{2} B_{3}} \beta^{*}\left(k_{1}\left(1-r_{1}\right)+B_{1}\right) B_{3} \sigma_{1}\left(1-r_{1}\right)\left(1-p_{1}\right) \\
& -2 \frac{\beta^{*} u_{3}^{2} v_{3} \mu}{B_{1}^{2} B_{3}} \gamma_{1} \beta^{*}\left(k_{1}\left(1-r_{1}\right) B_{3}+\delta_{1} B_{1}\right) B_{1} \sigma_{3}\left(1-\delta_{1}\right)
\end{aligned}
$$

so that the bifurcation coefficient $a>0$ if and only if

$$
\sigma_{1}>\frac{\left[p_{1} \mu+k_{1}\left(1-r_{1}\right)\right]\left[B_{3}\left(\beta^{*}\left(1-p_{1}\right)+B_{1}\right)+2 \gamma_{1} B_{1}\right]+2 \gamma_{1} \beta^{*}\left(k_{1}\left(1-r_{1}\right) B_{3}+\delta_{1} B_{1}\right) B_{1} \sigma_{3}\left(1-\delta_{1}\right)}{2 \beta^{*}\left(k_{1}\left(1-r_{1}\right)+B_{1}\right) B_{3}\left(1-r_{1}\right)\left(1-p_{1}\right)} .
$$

Thus, $b>0$ and $a>0$ or $a<0$ depending on whether inequality (3.16) is satisfied. This sign of $b$ may be expected in general for epidemic models because, in essence, using $\beta$ as a bifurcation parameter often ensures $b>0$. Using Theorem 3 items (i) and (iv), we establish the following result.

Theorem 3.5. If condition (3.16) is satisfied, $a>0$, then, model system (2.3) without DM undergoes a backward bifurcation at $\mathcal{R}_{0}^{T}=1$, otherwise $a<0$ and a unique endemic equilibrium $Q^{*}$ guaranteed by Lemma 3.3 is locally asymptotically stable for $\mathcal{R}_{0}^{T}>1$, but close to 1 .

The phenomenon of backward bifurcation in disease models, where a stable endemic equilibrium coexists with a stable disease-free equilibrium when the associated reproduction number is less than the unity, has important implications for disease control [46]. In such a scenario, the classical requirement of the basic reproduction number being less than the unity becomes only a necessary, but not sufficient condition for disease elimination. For $a<0$, the model exhibits a forward bifurcation. The bifurcations which occur for different signs of $a$ are shown in Fig. 4.

\subsubsection{Co-existence of non-diabetics and diabetics endemic equilibrium}

System (2.4) can be rewritten in the following compact form:

$$
\left\{\begin{array}{l}
x^{\prime}(t)=\Gamma+A_{x} x(t)-\lambda \mathcal{H}_{1} x(t), \\
y^{\prime}(t)=\lambda\left[\mathcal{H}_{2} x(t)+\mathcal{H}_{3} y(t)\right]+A_{y} y(t),
\end{array}\right.
$$

where $x(t) \in \mathbb{R}_{+}^{2}, y(t) \in \mathbb{R}_{+}^{6}, \Gamma, \lambda, B, A_{x}$ and $A_{y}$ are defined as in Eq. (2.4). In Eq. (3.17), the matrices $\mathcal{H}_{1}, \mathcal{H}_{2}$ and $\mathcal{H}_{3}$ are defined as 

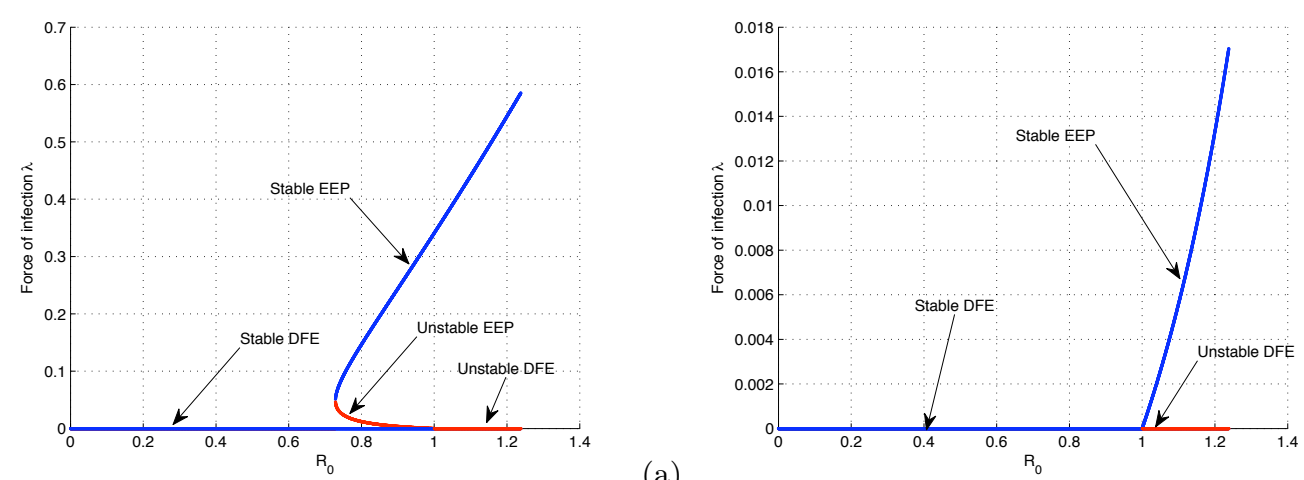

(a)

Figure 4. Bifurcation diagram for model system (2.3) without DM. (a) $\sigma_{1}=0.1$ (so that $a>0$ ) and (b) $\sigma_{1}=0.70 * p_{1}$ (so that $a<0$ ). The notation EEP stands for endemic equilibrium point. All other parameter as in Table 1.

$$
\mathcal{H}_{1}=\left(\begin{array}{ll}
1 & 0 \\
0 & \theta
\end{array}\right), \quad \mathcal{H}_{2}=\left(\begin{array}{cc}
1-p_{1} & 0 \\
p_{1} & 0 \\
0 & 0 \\
0 & 1-p_{2} \\
0 & p_{2} \\
0 & 0
\end{array}\right)
$$

and

$$
\mathcal{H}_{3}=\left(\begin{array}{cccccc}
-\sigma_{1}\left(1-r_{1}\right) & 0 & \sigma_{3}\left(1-\delta_{1}\right) & 0 & 0 & 0 \\
\sigma_{1}\left(1-r_{1}\right) & 0 & 0 & 0 & 0 & 0 \\
0 & 0 & -\sigma_{3}\left(1-\delta_{1}\right) & 0 & 0 & 0 \\
0 & 0 & 0 & -\theta \sigma_{2}\left(1-r_{1}\right) & 0 & \theta \sigma_{4}\left(1-\delta_{2}\right) \\
0 & 0 & 0 & \theta \sigma_{2}\left(1-r_{1}\right) & 0 & 0 \\
0 & 0 & 0 & 0 & 0-\theta \sigma_{4}\left(1-\delta_{2}\right)
\end{array}\right) .
$$

The basic reproduction number of model system (3.17) is now given by

$$
\mathcal{R}_{0}=\frac{1}{N_{0}} \sum_{i=1}^{2} S_{i}^{0}\left\langle B \mid\left(-A_{y}^{-1}\right) \mathcal{H}_{2}\right\rangle,
$$

where $N_{0}=\Lambda / \mu$.

Let $Q^{* *}=\left(x^{* *}, y^{* *}\right)$ be the positive endemic equilibrium of model system (3.17). This positive endemic equilibrium (steady state with $y(t)>0$ ) is obtained by setting the right hand side of model system (3.17) to zero, giving:

$$
\left\{\begin{array}{l}
\Gamma+\left(A_{x}-\lambda^{* *} \mathcal{H}_{1}\right) x^{* *}=0, \\
\lambda^{* *} \mathcal{H}_{2} x^{* *}+\left(A_{y}+\lambda^{* *} \mathcal{H}_{3}\right) y^{* *}=0,
\end{array}\right.
$$

where

$$
\lambda^{* *}=\frac{\left\langle B \mid y^{* *}\right\rangle}{N^{* *}},
$$

is the force of infection at the endemic equilibrium.

The following result have been established in Appendix.

Lemma 3.6. The TB model (2.3)

(i) could have a unique endemic equilibrium whenever $\mathcal{R}_{0}>1$. 
(ii) could have more than one endemic equilibrium whenever $\mathcal{R}_{0}>1$.

(iii) could have a unique endemic equilibrium whenever $\mathcal{R}_{0}<1$.

(iv) could have one or more endemic equilibria whenever $\mathcal{R}_{0}<1$.

The backward bifurcation phenomenon is illustrated by simulating model system (2.3) with the parameter values of Table 1 . A time series of the model system $(2.3)$ when $\beta=7$ (so that $\mathcal{R}_{0}=0.8597$ ) is shown in Fig. 5. This figure shows the convergence to both the disease-free equilibrium and the endemic equilibrium for model system $(2.3)$ when $\mathcal{R}_{0}<1$. This illustrates that the profiles can converge to either the disease free equilibrium or an endemic equilibrium point, depending on the initial sizes of the population of the model (owing to the phenomenon of backward bifurcation).

\section{Numerical studies}

Numerical simulations using a set of reasonable parameter values in Table 1 are carried out for illustrative purpose and to support the analytical results. In all simulations, the model was simulated with the following initial conditions which have been chosen arbitrarily: $S_{1}(0)=8741400, S_{2}(0)=200000$, $E_{1}(0)=557800, E_{2}(0)=4500, I_{1}(0)=20000, I_{2}(0)=1800, R_{1}(0)=8000$ and $R_{2}(0)=200$. In all simulations, the transmission rate $\beta$ has been chosen such that $\mathcal{R}_{0}>1$ (so that case (i) of Lemma 3 is satisfied).

\subsection{General dynamics}

Numerical simulations of model system (2.3) showing the time series plots of non-diabetics and diabetics infective populations when $\beta=10$ (so that $\mathcal{R}_{0}=1.7194$ ) are shown in Fig. 6 . The results obtained using parameter values in Table 1 indicate that more diabetics $\left(E_{2}, I_{2}\right)$ are infected than non-diabetics $\left(E_{1}, I_{1}\right)$. The result is in agreement with various studies which suggested that DM increases the number of infected TB individuals in the community [11-16]. This result suggests that DM can cause serious problems for someone with TB. Thus, consideration of how lessons learned from management of TB as a chronic illness could be applied to DM management in resource-poor settings is worthy of greater exploration, aiming to improve diabetes chronic disease management and consequently outcomes of both diseases. It is therefore imperative to screen diabetics for TB and TB patients for DM.

\subsection{Effects of increased the susceptibility to TB du to DM}

Effects of increased the susceptibility to TB as a result of DM are explored in Fig. 7 by varying the parameter $\theta$ when $\beta=9$ (so that $\mathcal{R}_{0}>1$ ). The numerical results in Fig. 7(a)-(d) illustrate that an increases of the susceptibility to TB due to DM will generally result in an increases of the fraction of TB infected individuals (both diabetics and non diabetics) with a significant effect on the diabetics (as shown by Figs. $7(\mathrm{~b})-(\mathrm{c}))$. The figure shows that the changes in $\theta$ do not significantly affect the long term progression of the disease, but significant changes are observed in the initial phases of the epidemic, with more changes being observed in the diabetics infective individuals.

\subsection{Effects of increased some parameters}

Simulation results in Fig. 8 illustrate the variation of the rate of being diabetics $\alpha$ on the dynamics of model system $(2.3)$ when $\beta=8$ (so that $\mathcal{R}_{0}>1$ ). This figures suggests that an increases of the recruitment of diabetics in the community will increase the prevalence of TB cases. This suggests that whenever more individuals in the community become hyperglycaemia, this may influence the spread of $\mathrm{TB}$ in the community, but if the reverse is true, with the basic reproduction number greater than the unity, then a number of factors may play a crucial role on the spread of TB. This result suggests that the problem of DM should be addressed in communities affected with TB in order to reduce the burden of the disease. 
Numerical results in Fig. 9 shows the role of $\tau$ on the dynamics of TB when $\beta=8$ (so that $\mathcal{R}_{0}>1$ ). From this figure, it clearly evident that the modification parameter $\tau$ does not have a significantly negative impact on the dynamical transmission of TB in a community.

\subsection{Effects of varying the percentage of heavy alcohol drinkers in the population}

Herein, we now demonstrate the effects of increasing the percentage of diabetics in the population. Figure 10 is a graphical representation of the total number of TB infected individuals who are nondiabetics and diabetics in the absence and presence of treatment using various initial conditions when the percentage of diabetics inside the population vary and $\beta=5$ (so that $\mathcal{R}_{0}>1$ ).

Figures 10(a) and (b) show what happens in a population with no treatment of infectious (i.e., $\gamma_{1}=$ $\gamma_{2}=0$ ), while Figs. 10 (c) and (d) show what happens in a population with treatment (i.e., $\gamma_{1}=0.7372$ and $\left.\gamma_{2}=0.7372\right)$ as the percentage of diabetics is varied.

This figure shows that as the percentage of diabetics in the population increases, the total number of TB infected individuals who are non-diabetics at the endemic equilibrium decreases (see Figs. 10(a) and (c)), while the total number of TB infected individuals who are diabetics at the endemic equilibrium incresases (see Figs. 10(b) and (d)). Also, from this figure, it cleary appears that the total number of TB infected individuals who are non-diabetics and diabetics at the endemic equilibrium in the presence of TB treatment is less than the total number of TB infected individuals who are non-diabetics and diabetics at the endemic equilibrium in the absence of TB treatment. Thus, TB treatment is able to effectively control the epidemic. However, it is worth noting that the rate of decreases is higher among TB patients who are non-diabetics than among the patients who are diabetics. This tends to suggest that DM negatively affects TB. A mere look at Fig. 10(d) shows that decreasing the percentage of diabetics to less than $50 \%$ in a country where there is effective TB treatment will have a positive impact on TB control as noted by a rapid depletion of TB infected individuals. However, when the percentage is $100 \%$ (the whole population is diabetics) as noted in Figs. 10(b) and (d), even if effective treatment is available TB cannot be managed. This means that DM can complicate the management of TB. This suggests that DM positively enhances TB infection and disease progression while negatively affecting treatment, implying it should be or has to be discouraged at all levels within the population if TB is to be eradicated.

\section{Discussions and concluding remarks}

The link between DM and TB has been recognized for centuries. In recent decades, tuberculosis incidence has declined in high-income countries, but incidence remains high in countries that have high rates of infection with HIV, high prevalence of malnutrition and crowded living conditions, or poor TB control infrastructure. At the same time, diabetes prevalence is soaring globally, fueled by obesity. There is growing evidence that DM is an important risk factor for TB and might affect disease propagation and treatment response. Furthermore, TB might induce glucose intolerance and worsen glycaemia control in people with diabetes. DM patients with poorer glycaemic control appear to be at higher risk for TB, demonstrating a dose-response relationship between the degree and duration of hyperglycaemia and vulnerability to TB, rather similar to that observed with HIV and TB.

A mathematical model for studies the dynamics of TB transmission in a community, taking into account the fact that some people in the population are diabetics is presented as a system of ordinary differential equations. The effect of DM on the actual TB dynamics is twofold: it increases the transmissibility from diabetics infected individuals, and it also increases the progression from a latent stage of TB to an active TB in the population of diabetics. We have argued that there is an important interaction between DM and TB, the effects of which may be similar to those observed between HIV and TB. Although at the individual level the risk of developing TB is considerably lower in persons with DM than in HIV patients, the much larger, and rapidly growing, pool of DM patients makes the global and population attributable fraction of TB due to DM very similar to that seen with HIV. 
A qualitative analysis of the model has been presented. The epidemic threshold parameter which determines the outcome of the disease is computed and used to assess the dynamics of the disease in the community. Results show that increasing effective contact rate to become diabetics and susceptibility to TB due to DM increases the basic reproduction number $\mathcal{R}_{0}$, which agrees with experimental results that DM is associated with specific negative health outcomes $[48,49]$ and promotes the development of TB [50]. The disease-free and endemic equilibria are obtained and their stabilities are investigated depending on the system parameters. Due to the presence of backward bifurcation, in some parameter regimes the system exhibits a bi-stability between a disease free and endemic steady states. The Centre Manifold theory was used to determine the local asymptotic stability of the endemic equilibrium.

Numerical results are performed to illustrate various dynamical regimes. Graphical representations clearly show that an increase of the susceptibility to TB due to DM will generally result in an increases in the number of TB infected individuals with more effect on latently infected individuals and infectious who are diabetics. Thus, DM increases disease transmission, and the prevalence of disease increases with increased the rate of becoming diabetics. Furthermore, in the presence of treatment, TB control is more effective in communities with non-diabetics than in the communities with diabetics. Thus, DM may reduce the effectiveness of TB treatment and enhances TB transmission and disease progression. Hence, DM negatively affects TB control and as long as this control is taken as a biomedical intervention only, chemoprophylaxis and treatment alone may not be successful in populations where DM is common. Care for TB patients involves more than just giving the anti-TB treatment, and if additional support (from detection or diagnosis) of disease to compliance and completion of the appropriate treatment regime) is not provided, an $88 \%$ cure rate as promote by DOTS program would not likely be reached. In the event, when we have more individuals becoming diabetics, there is urgent need for intervention strategies such as counseling and educational campaigns in order to curtail the TB spread. Indeed, with a global DM epidemic that is escalating rapidly, the prevention and control of DM is likely to be an equally important intervention in controlling the DM-TB epidemic. Primary prevention of DM through attention to unhealthy diets, sedentary lifestyles and childhood and adult obesity must be included in broad prevention strategies for non-communicable diseases such as TB. Better integration of communicable and non-communicable disease prevention and care strategies that are focused on the patient rather than the disease is required. As with TB-HIV, one can adapt and apply similar methods of preventing, screening and treating the two diseases together, and ensure that we have secure pipelines for drugs, that we develop mechanisms of working together, that we undertake relevant operational and other kinds of research, and that we adopt the philosophy of 'learning by doing'. We may then stand a good chance of winning this war.

Appendix: Co-existence of non-diabetics and diabetics endemic equilibrium of model system (3.17)

Let $Q^{* *}=\left(x^{* *}, y^{* *}\right)$ be the positive endemic equilibrium of model system (3.17). This positive endemic equilibrium (steady state with $y(t)>0$ ) is obtained by setting the right hand side of model system (3.17) to zero, giving:

$$
\left\{\begin{array}{l}
\Gamma+\left(A_{x}-\lambda^{* *} \mathcal{K}_{1}\right) x^{* *}=0 \\
\lambda^{* *} \mathcal{K}_{2} x^{* *}+\left(A_{y}+\lambda^{* *} \mathcal{K}_{3}\right) y^{* *}=0
\end{array}\right.
$$

where

$$
\lambda^{* *}=\frac{\left\langle B \mid y^{* *}\right\rangle}{N^{* *}},
$$

is the force of infection at the endemic equilibrium. Using the first equation of (A.1), one has

$$
S_{1}^{* *}=\frac{\Lambda}{\left(\mu+\alpha+\lambda^{* *}\right)} \quad \text { and } \quad S_{2}^{* *}=\frac{\alpha \Lambda}{\left(\mu+\theta \lambda^{* *}\right)\left(\mu+\alpha+\lambda^{* *}\right)} .
$$

Since $y^{* *} \geq 0,\left(A_{y}+\lambda^{* *} \mathcal{K}_{3}\right)$ is a Metzler matrix [41,42] and one can deduce that $-\left(A_{y}+\lambda^{* *} \mathcal{K}_{3}\right)^{-1} \geq 0$. With this in mind, multiplying the second equation of Eq. (A.1) by $-\left(A_{y}+\lambda^{* *} \mathcal{K}_{1}\right)^{-1}$ yields

$$
y^{* *}=\lambda^{* *}\left(-\left(A_{y}+\lambda^{* *} \mathcal{K}_{3}\right)\right)^{-1} \mathcal{K}_{2} x^{* *} .
$$


From Eq. (A.4), one has

$$
\begin{aligned}
& E_{1}^{* *}=\frac{S_{1}^{* *} \lambda^{* *}\left(a_{3}+a_{4} \lambda^{* *}\right)}{b_{2}\left(\lambda^{* *}\right)^{2}+b_{1} \lambda^{* *}+b_{0}}, \quad I_{1}^{* *}=\frac{S_{1}^{* *} \lambda^{* *}\left[a_{5}+a_{6} \lambda^{* *}+a_{7}\left(\lambda^{* *}\right)^{2}\right]}{b_{2}\left(\lambda^{* *}\right)^{2}+b_{1} \lambda^{* *}+b_{0}}, \\
& R_{1}^{* *}=\frac{\gamma_{2} S_{1}^{* *} \lambda^{* *}\left(a_{8}+a_{9} \lambda^{* *}\right)}{b_{2}\left(\lambda^{* *}\right)^{2}+b_{1} \lambda^{* *}+b_{0}}, \\
& E_{2}^{* *}=\frac{\alpha S_{1}^{* *} \lambda^{* *}\left[a_{10}+a_{11} \lambda^{* *}+a_{12}\left(\lambda^{* *}\right)^{2}+a_{13}\left(\lambda^{* *}\right)^{3}+a_{14}\left(\lambda^{* *}\right)^{4}+a_{15}\left(\lambda^{* *}\right)^{5}\right]}{\left(\mu+\theta \lambda^{* *}\right)\left[a_{2}\left(\lambda^{* *}\right)^{2}+a_{1} \lambda^{* *}+a_{0}\right]\left[b_{2}\left(\lambda^{* *}\right)^{2}+b_{1} \lambda^{* *}+b_{0}\right]}, \\
& I_{2}^{* *}=\frac{\alpha S_{1}^{* *} \lambda^{* *}\left[a_{16}+a_{17}\left(\lambda^{* *}\right)+a_{18}\left(\lambda^{* *}\right)^{2}+a_{19}\left(\lambda^{* *}\right)^{3}+a_{20}\left(\lambda^{* *}\right)^{4}+a_{21}\left(\lambda^{* *}\right)^{5}\right]}{\left(\mu+\theta \lambda^{* *}\right)\left[a_{2}\left(\lambda^{* *}\right)^{2}+a_{1} \lambda^{* *}+a_{0}\right]\left[b_{2}\left(\lambda^{* *}\right)^{2}+b_{1} \lambda^{* *}+b_{0}\right]}, \\
& R_{1}^{* *}=\frac{\alpha \lambda^{* *} S_{1}^{* *}\left[a_{22}+a_{23} \lambda^{* *}+a_{24}\left(\lambda^{* *}\right)^{2}+a_{25}\left(\lambda^{* *}\right)^{3}+a_{26}\left(\lambda^{* *}\right)^{4}\right]}{\left(\mu+\theta \lambda^{* *}\right)\left[a_{2}\left(\lambda^{* *}\right)^{2}+a_{1} \lambda^{* *}+a_{0}\right]\left[b_{2}\left(\lambda^{* *}\right)^{2}+b_{1} \lambda^{* *}+b_{0}\right]},
\end{aligned}
$$

where

$$
\begin{aligned}
& a_{0}=A_{1}\left(A_{2} A_{3}-\gamma_{1} \delta_{1}\right), a_{1}=\sigma_{1}\left(1-r_{1}\right)\left(A_{2} A_{3}-\gamma_{1} \delta_{1}\right)+\sigma_{3}\left(A_{1} A_{2}-\gamma_{1} k_{1}\left(1-r_{1}\right)\right), \\
& a_{2}=\sigma_{1}\left(1-r_{1}\right) \sigma_{3}\left(1-\delta_{1}\right)\left(A_{2}-\gamma_{1}\right), \quad b_{2}=\theta^{2} \sigma_{2}\left(1-r_{2}\right) \sigma_{4}\left(1-\delta_{2}\right)\left(A_{5}-\gamma_{2}\right), \\
& b_{1}=\theta\left(\sigma_{4}\left(1-\delta_{2}\right)\left(A_{4} A_{5}-\gamma_{2} k_{2}\left(1-r_{2}\right)\right)+\sigma_{2}\left(A_{5} A_{6}-\gamma_{2} \delta_{2}\right)\right), b_{0}=\left(A_{5} A_{6}-\gamma_{2} \delta_{2}\right) A_{4}, \\
& a_{3}=\left(A_{5} A_{6}-\gamma_{2} \delta_{2}\right)\left(1-p_{1}\right), \\
& a_{4}=\theta \sigma_{4}\left(1-\delta_{2}\right)\left(\gamma_{2} p_{1}+A_{5}\left(1-p_{1}\right)\right), \\
& a_{5}=A_{6}\left(A_{4} p_{1}+k_{2}\left(1-r_{2}\right)\left(1-p_{1}\right)\right), \\
& a_{6}=\theta \sigma_{4}\left(1-\delta_{2}\right)\left(A_{4} p_{1}+k_{2}\left(1-r_{2}\right)\left(1-p_{1}\right)\right)+\theta \sigma_{2}\left(1-r_{2}\right) A_{6}, \\
& a_{7}=\theta \sigma_{4}\left(1-\delta_{2}\right) \theta \sigma_{2}\left(1-r_{2}\right), \\
& a_{8}=A_{4} p_{1}+k_{2}\left(1-r_{2}\right)\left(1-p_{1}\right), \\
& a_{9}=\theta \sigma_{2}\left(1-r_{2}\right),
\end{aligned}
$$

$$
\begin{aligned}
a_{10} & =\mu c_{00}\left(1-p_{1}\right)+\left(A_{2} A_{3}-\gamma_{1} \delta_{1}\right) b_{0}\left(1-p_{2}\right), \\
a_{11} & =\mu p_{1} c_{10}+\left(\theta c_{00}+\mu c_{01}\right)\left(1-p_{1}\right)+\gamma_{1} \sigma_{3} b_{0} p_{2}+\left(\left(A_{2} A_{3}-\gamma_{1} \delta_{1}\right) b_{1}+\sigma_{3} A_{2} b_{0}\right)\left(1-p_{2}\right), \\
a_{12} & =p_{1}\left(\theta c_{10}+\mu c_{11}\right)+\left(1-p_{1}\right)\left(\theta c_{01}+\mu c_{02}\right)+\gamma_{1} \sigma_{3}\left(1-\delta_{1}\right) b_{1} p_{2}+\left(\left(A_{2} A_{3}-\gamma_{1} \delta_{1}\right) b_{2}\right. \\
& \left.+\sigma_{3}\left(1-\delta_{1}\right) A_{2} b_{1}\right)\left(1-p_{2}\right), \\
a_{13} & =p_{1}\left(\theta c_{11}+\mu c_{12}\right)+\left(\theta c_{02}+\mu c_{03}\right)\left(1-p_{1}\right)+\gamma_{1} \sigma_{3}\left(1-\delta_{1}\right) b_{2} p_{2}+\sigma_{3}\left(1-\delta_{1}\right) A_{2} b_{2}\left(1-p_{2}\right), \\
a_{14} & =\left(\theta c_{12}+\mu c_{13}\right) p_{1}+\theta c_{03}\left(1-p_{1}\right), \\
a_{15} & =\theta p_{1} c_{13}, \\
a_{16} & =\mu p_{1} d_{10}+\mu d_{00}\left(1-p_{1}\right)+A_{1} A_{3} b_{0} p_{2}+A_{3} b_{0} k_{1}\left(1-r_{1}\right)\left(1-p_{2}\right), \\
a_{17} & =p_{1}\left(\theta d_{10}+\mu d_{11}\right)+\left(1-p_{1}\right)\left(\theta d_{00}+\mu d_{01}\right)+p_{2}\left(\sigma_{1}\left(1-r_{1}\right) A_{3} b_{0}+\sigma_{3} A_{1} b_{0}+A_{1} A_{3} b_{1}\right) \\
& +\left(1-p_{2}\right)\left(\sigma_{1}\left(1-r_{1}\right) A_{3} b_{0}+\lambda \sigma_{3} b_{0} k_{1}\left(1-r_{1}\right)+A_{3} b_{1} k_{1}\left(1-r_{1}\right)\right),
\end{aligned}
$$




$$
\begin{aligned}
a_{18} & =p_{1}\left(\theta d_{11}+\mu d_{12}\right)+p_{2}\left(\sigma_{1}\left(1-r_{1}\right) \sigma_{3}\left(1-\delta_{1}\right) b_{0}+\sigma_{1}\left(1-r_{1}\right) A_{3} b_{1}+\sigma_{3} A_{1} b_{1}+A_{1} A_{3} b_{2}\right) \\
& +\left(1-p_{2}\right)\left(\sigma_{1}\left(1-r_{1}\right) \sigma_{3}\left(1-\delta_{1}\right) b_{0}+\sigma_{1} A_{3} b_{1}+\sigma_{3}\left(1-\delta_{1}\right) b_{1} k_{1}\left(1-r_{1}\right)+A_{3} b_{2} k_{1}\left(1-r_{1}\right)\right) \\
& +\left(1-p_{1}\right)\left(\theta d_{01}+\mu d_{02}\right), \\
a_{19} & =p_{1}\left(\theta d_{12}+\mu d_{13}\right)+\left(1-p_{1}\right)\left(\theta d_{02}+\mu d_{03}\right)+\left(1-p_{2}\right)\left(\sigma_{3}\left(1-\delta_{1}\right) b_{2} k_{1}\left(1-r_{1}\right)+\sigma_{1}\left(1-r_{1}\right) A_{3} b_{2}\right) \\
& +p_{2}\left(\sigma_{1}\left(1-r_{1}\right) \sigma_{3}\left(1-\delta_{1}\right) b_{1}+\sigma_{1}\left(1-r_{1}\right) \sigma_{3}\left(1-\delta_{1}\right) b_{1}+\sigma_{1} A_{3} b_{2}+\sigma_{3}\left(1-\delta_{1}\right) A_{1} b_{2}\right), \\
a_{20} & =p_{1}\left(\theta d_{13}+\mu d_{14}\right)+\left(1-p_{1}\right)\left(\mu d_{04}+\theta d_{03}\right)+\sigma_{1}\left(1-r_{1}\right) \sigma_{3}\left(1-\delta_{1}\right) b_{2} p_{2}+\sigma_{1}\left(1-r_{1}\right) \sigma_{3}\left(1-\delta_{1}\right) b_{2}\left(1-p_{2}\right) \\
a_{21} & =\theta p_{1} d_{14}+\theta d_{04}\left(1-p_{1}\right), \\
a_{22} & =\mu e_{10} p_{1}+\mu e_{00}\left(1-p_{1}\right)+\gamma_{1} A_{1} b_{0} p_{2}+\gamma_{1} b_{0} k_{1}\left(1-r_{1}\right)\left(1-p_{2}\right), \\
a_{23} & =p_{1}\left(\theta e_{10}+\mu e_{11}\right)+\left(1-p_{1}\right)\left(\theta e_{00}+\mu e_{01}\right)+p_{2}\left(\sigma_{1}\left(1-r_{1}\right) \gamma_{1} b_{0}+\lambda \gamma_{1} A_{1} b_{1}\right) \\
& +\left(1-p_{2}\right)\left(\sigma_{1}\left(1-r_{1}\right) \gamma_{1} b_{0}+\gamma_{1} b_{1} k_{1}\left(1-r_{1}\right)\right), \\
a_{24} & =p_{1}\left(\theta e_{11}+\mu e_{12}\right)+\left(1-p_{1}\right)\left(\mu e_{02}+\theta e_{01}\right)+p_{2}\left(\sigma_{1}\left(1-r_{1}\right) \gamma_{1} b_{1}+\gamma_{1} A_{1} b_{2}\right) \\
& +\left(1-p_{2}\right)\left(\sigma_{1}\left(1-r_{1}\right) \gamma_{1} b_{1}+\gamma_{1} b_{2} k_{1}\left(1-r_{1}\right)\right), \\
a_{25} & =\left(\theta e_{12}+\mu e_{13}\right) p_{1}+\left(\theta e_{02}+\mu e_{03}\right)\left(1-p_{1}\right)+\sigma_{1}\left(1-r_{1}\right) \gamma_{1} b_{2} p_{2}+\sigma_{1}\left(1-r_{1}\right) \gamma_{1} b_{2}\left(1-p_{2}\right), \\
a_{26} & =\theta e_{13} p_{1}+\theta e_{03}\left(1-p_{1}\right),
\end{aligned}
$$

with

$$
\begin{aligned}
c_{00} & =\left(A_{5} A_{6}-\gamma_{2} \delta_{2}\right)\left(A_{2} A_{3}-\gamma_{1} \delta_{1}\right), \\
c_{01} & =\sigma_{3}\left(1-\delta_{1}\right) A_{2}\left(\gamma_{2} k_{2}\left(1-r_{2}\right)-\delta_{2} A_{2}\right)+\sigma_{3} A_{6}\left(\tau \gamma_{1} k_{2}\left(1-r_{2}\right)+A_{2} A_{5}\right)+\left(A_{2} A_{3}-\gamma_{1} \delta_{1}\right) A_{5} \theta \sigma_{4}\left(1-\delta_{2}\right), \\
c_{02} & =\theta \sigma_{2}\left(1-r_{2}\right) \sigma_{3}\left(1-\delta_{1}\right) A_{2}\left(\gamma_{2}+A_{5}\right)+\theta \tau \gamma_{1} \sigma_{3}\left(1-\delta_{1}\right)\left(\sigma_{2}\left(1-r_{2}\right) A_{6}+\sigma_{3}\left(1-\delta_{1}\right) k_{2}\left(1-r_{2}\right)\right), \\
c_{03} & =\theta^{2} \tau \sigma_{2}\left(1-r_{2}\right) \gamma_{1} \sigma_{3}\left(1-\delta_{1}\right) \sigma_{4}\left(1-\delta_{2}\right), \\
d_{00} & =\left(A_{5} A_{6}-\gamma_{2} \delta_{2}\right) A_{3} k_{1}\left(1-r_{1}\right)+A_{1} k_{2}\left(1-r_{2}\right)\left(\gamma_{2} \delta_{1}+\tau A_{3} A_{6}\right), \\
d_{01} & =\sigma_{1}\left(1-r_{1}\right) \gamma_{2}\left(\delta_{1} k_{2}\left(1-r_{2}\right)-\delta_{2} A_{3}\right)+\sigma_{3} \gamma_{2} k_{1}\left(1-r_{1}\right)\left(k_{2}\left(1-r_{2}\right)-\delta_{2}\right) \\
& +\sigma_{1}\left(1-r_{1}\right) A_{3} A_{6}\left(A_{5}+\tau k_{2}\left(1-r_{2}\right)\right)+\sigma_{3}\left(1-\delta_{1}\right) A_{6}\left(A_{5} k_{1}\left(1-r_{1}\right)+\tau A_{1} k_{2}\left(1-r_{2}\right)\right) \\
& +\theta \sigma_{2} A_{1}\left(\gamma_{2} \delta_{1}+\tau A_{3} A_{6}\right)+\theta \sigma_{4} A_{3}\left(A_{5} k_{1}\left(1-r_{1}\right)+\tau A_{1} k_{2}\left(1-r_{2}\right)\right),
\end{aligned}
$$

$$
\begin{aligned}
d_{02} & =\sigma_{1}\left(1-r_{1}\right) \sigma_{3}\left(1-\delta_{1}\right)\left(-\gamma_{2} \delta_{2}+A_{5} A_{6}+\left(\gamma_{2}+\tau A_{6}\right) k_{2}\left(1-r_{2}\right)\right)+\theta^{2} \tau \sigma_{2} \sigma_{4}\left(1-\delta_{2}\right) A_{1} A_{3} \\
& +\sigma_{1}\left(1-r_{1}\right) \sigma_{2}\left(1-r_{2}\right) \theta\left(\gamma_{2} \delta_{1}+\tau A_{3} A_{6}\right)+\sigma_{2}\left(1-r_{2}\right) \sigma_{3}\left(1-\delta_{1}\right) \theta\left(\gamma_{2} k_{1}\left(1-r_{1}\right)\right. \\
& \left.+\tau A_{1} A_{6}\right)+\theta \sigma_{1}\left(1-r_{1}\right) \sigma_{4}\left(1-\delta_{2}\right)\left(A_{3} A_{5}+\tau A_{3} k_{2}\left(1-r_{2}\right)\right) \\
& +\theta \sigma_{3}\left(1-\delta_{1}\right) \sigma_{4}\left(1-\delta_{2}\right)\left(A_{5} k_{1}\left(1-r_{1}\right)+\tau A_{1} k_{2}\left(1-r_{2}\right)\right) \\
d_{03} & =\theta^{2} \tau \sigma_{4}\left(1-\delta_{2}\right) \sigma_{2}\left(1-r_{2}\right)\left(\sigma_{1}\left(1-r_{1}\right) A_{3}+\sigma_{3} A_{1}\right) \\
& +\theta \tau \sigma_{1}\left(1-r_{1}\right) \sigma_{3}\left(1-\delta_{1}\right)\left(\sigma_{2}\left(1-r_{2}\right) A_{6}+\tau \sigma_{4} k_{2}\left(1-r_{2}\right)\right) \\
& +\theta \sigma_{1}\left(1-r_{1}\right) \sigma_{3}\left(1-\delta_{1}\right)\left(\sigma_{2}\left(1-r_{2}\right) \gamma_{2}+\sigma_{4} A_{5}\right),
\end{aligned}
$$

$$
\begin{aligned}
d_{04} & =\theta^{2} \tau \sigma_{1}\left(1-r_{1}\right) \sigma_{2}\left(1-r_{2}\right) \sigma_{3}\left(1-\delta_{1}\right) \sigma_{4}\left(1-\delta_{2}\right) \\
e_{00} & =A_{1} k_{2}\left(1-r_{2}\right)\left(\gamma_{2} A_{2}+\tau \gamma_{1} k_{2}\left(1-r_{2}\right)\right)+\gamma_{1} k_{1}\left(1-r_{1}\right)\left(A_{5} A_{6}-\gamma_{2} \delta_{2}\right) \\
e_{01} & =\sigma_{1}\left(1-r_{1}\right) \gamma_{1}\left(A_{5} A_{6}-\gamma_{2} \delta_{2}\right)+\sigma_{1} k_{2}\left(1-r_{2}\right)\left(\gamma_{2} A_{2}+\tau \gamma_{1} A_{6}\right)+\theta A_{1} \sigma_{2}\left(\gamma_{2} A_{2}+\tau \gamma_{1} A_{6}\right) \\
& +\theta \gamma_{1} \sigma_{4}\left(A_{5} k_{1}\left(1-r_{1}\right)+\tau A_{1} k_{2}\left(1-r_{2}\right)\right), \\
e_{02} & =\theta \sigma_{1}\left(1-r_{1}\right) \sigma_{2}\left(1-r_{2}\right)\left(\gamma_{2} A_{2}+\tau \gamma_{1} A_{6}\right)+\theta \sigma_{1}\left(1-r_{1}\right) \sigma_{4}\left(1-\delta_{2}\right) \gamma_{1}\left(A_{5}+\tau k_{2}\left(1-r_{2}\right)\right) \\
& +\theta^{2} \tau \sigma_{2}\left(1-r_{2}\right) \gamma_{1} \sigma_{4}\left(1-\delta_{2}\right) A_{1},
\end{aligned}
$$




$$
\begin{aligned}
& e_{03}=\theta^{2} \tau \sigma_{1}\left(1-r_{1}\right) \sigma_{2}\left(1-r_{2}\right) \gamma_{1} \sigma_{4}\left(1-\delta_{2}\right) \\
& c_{10}=\sigma_{3}\left(1-\delta_{1}\right) \gamma_{2} A_{2} A_{4}+\theta \gamma_{2} \sigma_{4} A_{2} A_{3}+\tau \gamma_{1} \sigma_{3}\left(1-\delta_{1}\right) A_{4} A_{6}-\theta \gamma_{1} \gamma_{2} \delta_{1} \sigma_{4}\left(1-\delta_{2}\right), \\
& c_{11}=\theta \sigma_{2}\left(1-r_{2}\right) \sigma_{3}\left(1-\delta_{1}\right) \gamma_{2} A_{2}+\theta \sigma_{3} \gamma_{2} \sigma_{4}\left(1-\delta_{2}\right) A_{2}+\theta \tau \sigma_{2}\left(1-r_{2}\right) \gamma_{1} \sigma_{3} A_{6} \\
& +\theta \tau \gamma_{1} \sigma_{3}\left(1-\delta_{1}\right) \sigma_{4}\left(1-\delta_{2}\right) A_{4} \\
& c_{12}=\theta^{2} \tau \sigma_{2}\left(1-r_{2}\right) \gamma_{1} \sigma_{3}\left(1-\delta_{1}\right) \sigma_{4}\left(1-\delta_{2}\right), d_{10}=A_{1} A_{4}\left(\gamma_{2} \delta_{1}+\tau A_{3} A_{6}\right), \\
& d_{11}=\left(\sigma_{1}\left(1-r_{1}\right) A_{4}+\theta \sigma_{2}\left(1-r_{2}\right) A_{1}\right)\left(\gamma_{2} \delta_{1}+\tau A_{3} A_{6}\right)+\sigma_{3}\left(1-\delta_{1}\right) A_{4}\left(\gamma_{2} k_{1}\left(1-r_{1}\right)+\tau A_{1} A_{6}\right) \\
& +\theta \sigma_{4}\left(1-\delta_{2}\right) A_{3}\left(\gamma_{2} k_{1}\left(1-r_{1}\right)+\tau A_{1} A_{4}\right) \text {, } \\
& d_{12}=\sigma_{1}\left(1-r_{1}\right) \sigma_{3}\left(1-\delta_{1}\right)\left(\gamma_{2} A_{4}+\tau A_{4} A_{6}\right)+\theta \sigma_{1}\left(1-r_{1}\right) \sigma_{2}\left(1-r_{2}\right)\left(\gamma_{2} \delta_{1}+\tau A_{3} A_{6}\right) \\
& +\theta \sigma_{1} \sigma_{4}\left(1-\delta_{2}\right) A_{3}\left(\gamma_{2}+\tau A_{4}\right)+\theta \sigma_{2}\left(1-r_{2}\right) \sigma_{3}\left(\gamma_{2} k_{1}\left(1-r_{1}\right)+\tau A_{1} A_{6}\right) \\
& +\theta \sigma_{3}\left(1-\delta_{1}\right) \sigma_{4}\left(1-\delta_{2}\right)\left(\gamma_{2} k_{1}\left(1-r_{1}\right)+\tau A_{1} A_{4}\right)+\theta^{2} \tau \sigma_{2}\left(1-r_{2}\right) \sigma_{4}\left(1-\delta_{2}\right) A_{1} A_{3}, \\
& d_{13}=\theta \sigma_{1}\left(1-r_{1}\right) \sigma_{2}\left(1-r_{2}\right) \sigma_{3}\left(1-\delta_{1}\right)\left(\gamma_{2}+\tau A_{6}\right)+\theta \sigma_{1}\left(1-r_{1}\right) \sigma_{3}\left(1-\delta_{1}\right) \sigma_{4}\left(1-\delta_{2}\right)\left(\gamma_{2}+\tau A_{4}\right) \\
& +\theta^{2} \tau \sigma_{2}\left(1-r_{2}\right) \sigma_{4}\left(1-\delta_{2}\right)\left(\sigma_{1}\left(1-r_{1}\right) A_{3}+\tau \sigma_{3} A_{1}\right) \\
& d_{14}=\theta^{2} \tau \sigma_{1}\left(1-r_{1}\right) \sigma_{2}\left(1-r_{2}\right) \sigma_{3}\left(1-\delta_{1}\right) \sigma_{4}\left(1-\delta_{2}\right), e_{10}=A_{1} A_{4}\left(\gamma_{2} A_{2}+\tau \gamma_{1} A_{6}\right) \text {, } \\
& e_{11}=\theta \gamma_{1} \sigma_{4}\left(1-\delta_{2}\right)\left(\gamma_{2} k_{1}\left(1-r_{1}\right)+\tau A_{1} A_{4}\right)+\theta \sigma_{2}\left(1-r_{2}\right) A_{1}\left(\gamma_{2} A_{2}+\tau \gamma_{1} A_{6}\right) \\
& +\sigma_{1}\left(1-r_{1}\right) A_{4}\left(\tau \gamma_{1} A_{6}+\gamma_{2} A_{2}\right) \\
& e_{12}=\theta \sigma_{1}\left(1-r_{1}\right) \gamma_{2}\left(\gamma_{1} \sigma_{4}\left(1-\delta_{2}\right)+\sigma_{2} A_{2}\right)+\theta \tau \sigma_{1}\left(1-r_{1}\right) \gamma_{1}\left(\sigma_{2}\left(1-r_{2}\right) A_{6}\right. \\
& \left.+\tau \sigma_{4} A_{4}\right)+\theta^{2} \tau \sigma_{2}\left(1-r_{2}\right) \gamma_{1} \sigma_{4}\left(1-\delta_{2}\right) A_{1}, e_{13}=\theta^{2} \tau \sigma_{1}\left(1-r_{1}\right) \sigma_{2}\left(1-r_{2}\right) \gamma_{1} \sigma_{4}\left(1-\delta_{2}\right), \\
& c_{20}=\sigma_{3}\left(1-\delta_{1}\right) A_{4}\left(A_{2} A_{5}+\tau \gamma_{1} \delta_{2}\right)+\theta \sigma_{4}\left(1-\delta_{2}\right) A_{5}\left(A_{2} A_{3}-\gamma_{1} \delta_{1}\right) \text {, } \\
& c_{21}=\theta \sigma_{2}\left(1-r_{2}\right) \sigma_{3}\left(1-\delta_{1}\right)\left(A_{2} A_{5}+\tau \gamma_{1} \delta_{2}\right)+\theta \sigma_{3}\left(1-\delta_{1}\right) \sigma_{4}\left(1-\delta_{2}\right)\left(A_{2} A_{5}+\tau \gamma_{1} k_{2}\left(1-r_{2}\right)\right) \text {, } \\
& c_{22}=\theta^{2} \tau \sigma_{2}\left(1-r_{2}\right) \gamma_{1} \sigma_{3}\left(1-\delta_{1}\right) \sigma_{4}\left(1-\delta_{2}\right), \quad d_{20}=A_{1} A_{4}\left(\delta_{1} A_{5}+\tau \delta_{2} A_{3}\right), \\
& d_{21}=\sigma_{1}\left(1-r_{1}\right) A_{4}\left(\delta_{1} A_{5}+\tau \delta_{2} A_{3}\right)+\sigma_{3} A_{4}\left(A_{5} k_{1}\left(1-r_{1}\right)+\tau \delta_{2} A_{1}\right)+\theta \sigma_{2}\left(1-r_{2}\right) A_{1}\left(\delta_{1} A_{5}+\tau \delta_{2} A_{3}\right) \\
& +\theta \sigma_{4}\left(1-\delta_{2}\right) A_{3}\left(A_{5} k_{1}\left(1-r_{1}\right)+\tau A_{3} k_{2}\left(1-r_{2}\right)\right) \text {, } \\
& d_{22}=\sigma_{1}\left(1-r_{1}\right) \sigma_{3}\left(1-\delta_{1}\right) A_{4}\left(A_{5}+\tau \delta_{2}\right)+\theta \sigma_{1}\left(1-r_{1}\right) \sigma_{2}\left(1-r_{2}\right)\left(\delta_{1} A_{5}+\tau \delta_{2} A_{3}\right) \\
& +\theta \sigma_{1}\left(1-r_{1}\right) \sigma_{4}\left(1-\delta_{2}\right) A_{3}\left(A_{5}+\tau k_{2}\left(1-r_{2}\right)\right)+\theta \sigma_{2}\left(1-r_{2}\right) \sigma_{3}\left(1-\delta_{1}\right)\left(A_{5} k_{1}\left(1-r_{1}\right)+\tau \delta_{2} A_{1}\right) \\
& +\theta \sigma_{3}\left(1-\delta_{1}\right) \sigma_{4}\left(A_{5} k_{1}\left(1-r_{1}\right)+\tau A_{1} k_{2}\left(1-r_{2}\right)\right)+\theta^{2} \tau \sigma_{2}\left(1-r_{2}\right) \sigma_{4} A_{1} A_{3}, \\
& d_{23}=\theta \sigma_{1}\left(1-r_{1}\right) \sigma_{2}\left(1-r_{2}\right) \sigma_{3}\left(1-\delta_{1}\right)\left(A_{5}+\tau \delta_{2}\right)+\theta \sigma_{1}\left(1-r_{1}\right) \sigma_{3}\left(1-\delta_{1}\right) \sigma_{4}\left(1-\delta_{2}\right)\left(A_{5}\right. \\
& \left.+\tau k_{2}\left(1-r_{2}\right)\right)+\theta^{2} \sigma_{2}\left(1-r_{2}\right) \sigma_{4}\left(1-\delta_{2}\right) \tau\left(\sigma_{1}\left(1-r_{1}\right) A_{3}+\sigma_{3}\left(1-\delta_{1}\right) A_{1}\right), \\
& d_{24}=\theta^{2} \tau \sigma_{1}\left(1-r_{1}\right) \sigma_{2}\left(1-r_{2}\right) \sigma_{3}\left(1-\delta_{1}\right) \sigma_{4}\left(1-\delta_{2}\right), \quad e_{20}=\left(A_{2} A_{5}+\tau \gamma_{1} \delta_{2}\right) A_{1} A_{4}, \\
& e_{21}=\sigma_{1}\left(1-r_{1}\right) A_{4}\left(A_{2} A_{5}+\tau \gamma_{1} \delta_{2}\right)+\theta \gamma_{1} \sigma_{4}\left(1-\delta_{2}\right)\left(A_{5} k_{1}\left(1-r_{1}\right)+\tau A_{1} k_{2}\left(1-r_{2}\right)\right) \\
& +\theta \sigma_{2}\left(1-r_{2}\right) A_{1}\left(A_{2} A_{5}+\tau \gamma_{1} \delta_{2}\right) \\
& e_{22}=\theta \sigma_{1}\left(1-r_{1}\right) \gamma_{1} \sigma_{4}\left(1-\delta_{2}\right)\left(A_{5}+\tau k_{2}\left(1-r_{2}\right)\right)+\theta \sigma_{1}\left(1-r_{1}\right) \sigma_{2}\left(1-r_{2}\right)\left(A_{2} A_{5}+\tau \gamma_{1} \delta_{2}\right) \\
& +\theta^{2} \tau \sigma_{2}\left(1-r_{2}\right) \gamma_{1} \sigma_{4}\left(1-\delta_{2}\right) A_{1}, \quad e_{23}=\theta^{2} \tau \sigma_{1}\left(1-r_{1}\right) \sigma_{2}\left(1-r_{2}\right) \gamma_{1} \sigma_{4}\left(1-\delta_{2}\right),
\end{aligned}
$$

From Eq. (A.4), one can deduce that

$$
\left\langle B \mid y^{* *}\right\rangle=\frac{\beta S_{1}^{* *} \lambda^{* *}\left[f_{5}\left(\lambda^{* *}\right)^{5}+f_{4}\left(\lambda^{* *}\right)^{4}+f_{3}\left(\lambda^{* *}\right)^{3}+f_{2}\left(\lambda^{* *}\right)^{2}+f_{1} \lambda^{* *}+f_{0}\right]}{\left(\mu+\theta \lambda^{* *}\right)\left[a_{2}\left(\lambda^{* *}\right)^{2}+a_{1} \lambda^{* *}+a_{0}\right]\left[b_{2}\left(\lambda^{* *}\right)^{2}+b_{1} \lambda^{* *}+b_{0}\right]},
$$


where

$$
\begin{aligned}
& f_{0}=\alpha \varepsilon a_{16}+\mu a_{0} a_{5}, \\
& f_{1}=\alpha \varepsilon a_{17}+\theta a_{0} a_{5}+\mu a_{0} a_{6}+\mu a_{1} a_{5}, \\
& f_{2}=\alpha \varepsilon a_{18}+\theta a_{0} a_{6}+\theta a_{1} a_{5}+\mu a_{0} a_{7}+\mu a_{1} a_{6}+\mu a_{2} a_{5}, \\
& f_{3}=\alpha \varepsilon a_{19}+\theta a_{0} a_{7}+\theta a_{1} a_{6}+\theta a_{2} a_{5}+\mu a_{1} a_{7}+\mu a_{2} a_{6}, \\
& f_{4}=\alpha \varepsilon a_{20}+\theta a_{1} a_{7}+\theta a_{2} a_{6}+\mu a_{2} a_{7}, f_{5}=\alpha \varepsilon a_{21}+\theta a_{2} a_{7} .
\end{aligned}
$$

Combining Eqs. (A.2) and (A.6) yields

$$
N^{* *}=\frac{\beta S_{1}^{* *}\left[f_{5}\left(\lambda^{* *}\right)^{5}+f_{4}\left(\lambda^{* *}\right)^{4}+f_{3}\left(\lambda^{* *}\right)^{3}+f_{2}\left(\lambda^{* *}\right)^{2}+f_{1} \lambda^{* *}+f_{0}\right]}{\left(\mu+\theta \lambda^{* *}\right)\left[a_{2}\left(\lambda^{* *}\right)^{2}+a_{1} \lambda^{* *}+a_{0}\right]\left[b_{2}\left(\lambda^{* *}\right)^{2}+b_{1} \lambda^{* *}+b_{0}\right]} .
$$

Now, using Eq. (2.8) at the steady state, one has

$$
\begin{aligned}
N^{* *} & =\frac{\Lambda}{\mu}\left(1-\frac{d_{1} \gamma_{2}\left(a_{8}+\lambda^{* *} a_{9}\right) \lambda^{* *}}{\left(b_{2}\left[\lambda^{* *}\right)^{2}+b_{1} \lambda^{* *}+b_{0}\right]\left(\mu+\alpha+\lambda^{* *}\right)}\right) \\
& -\frac{\Lambda}{\mu} \frac{\lambda^{* *}\left[d_{2} \alpha\left(a_{22}+a_{23} \lambda^{* *}+a_{24}\left(\lambda^{* *}\right)^{2}+a_{25}\left(\lambda^{* *}\right)^{3}+a_{26}\left(\lambda^{* *}\right)^{4}\right)\right]}{\left(\mu+\theta \lambda^{* *}\right)\left[a_{2}\left(\lambda^{* *}\right)^{2}+a_{1} \lambda^{* *}+a_{0}\right]\left[b_{2}\left(\lambda^{* *}\right)^{2}+b_{1} \lambda^{* *}+b_{0}\right]\left(\mu+\alpha+\lambda^{* *}\right)}
\end{aligned}
$$

Equaling Eqs. (A.7) and (A.8), and using Eqs. (A.3), it can be shown that the non-zero equilibria of model system (3.17) satisfy the following sixth equation in $\lambda^{* *}$ :

$$
g_{6}\left(\lambda^{* *}\right)^{6}+g_{5}\left(\lambda^{* *}\right)^{5}+g_{4}\left(\lambda^{* *}\right)^{4}+g_{3}\left(\lambda^{* *}\right)^{3}+g_{2}\left(\lambda^{* *}\right)^{2}+g_{1} \lambda^{* *}+g_{0}=0,
$$

where

$$
\begin{aligned}
g_{0} & =\beta \mu f_{0}+\mu(\mu+\alpha) a_{0} b_{0} \\
g_{1} & =\beta \mu f_{1}+\mu a_{0} b_{0}+\theta(\mu+\alpha) a_{0} b_{0}+\mu(\mu+\alpha)\left(a_{0} b_{1}+a_{1} b_{0}\right)-\alpha d_{2} a_{22}-\mu \gamma_{2} a_{0} d_{1} a_{8}, \\
g_{2} & =\beta \mu f_{2}+\theta a_{0} b_{0}+\mu\left(a_{0} b_{1}+a_{1} b_{0}\right)+\theta(\mu+\alpha)\left(a_{1}\left(b_{0}+b_{1}\right)+a_{0}\left(b_{1}+b_{2}\right)+a_{2} b_{0}\right) \\
& -\theta \gamma_{2} d_{1} a_{0}\left(a_{0} a_{8}+\mu\left(a_{0} a_{9}+a_{1} a_{8}\right)\right)-\alpha d_{2} a_{23}, \\
g_{3} & =\beta \mu f_{3}+\theta\left(a_{0} b_{1}+a_{1} b_{0}\right)+\mu\left(a_{0} b_{2}+a_{1} b_{1}+a_{2} b_{0}\right)+\theta(\mu+\alpha)\left(a_{0} b_{2}+a_{1} b_{1}+a_{2} b_{0}\right), \\
& +\mu(\mu+\alpha)\left(a_{1} b_{2}+a_{2} b_{1}\right)-\theta \gamma_{2} d_{1}\left(a_{0} a_{9}+a_{1} a_{8}\right)-\alpha d_{2} a_{24}-\mu \gamma_{2} d_{1}\left(a_{1} a_{9}+a_{2} a_{8}\right), \\
g_{4} & =\beta \mu f_{4}+\theta\left(a_{0} b_{2}+a_{1} b_{1}+a_{2} b_{0}\right)+\mu\left(a_{1} b_{2}+a_{2} b_{1}\right)+\theta(\mu+\alpha)\left(a_{1} b_{2}+a_{2} b_{1}\right) \\
& +\mu(\mu+\alpha) a_{2} b_{2}-\theta \gamma_{2} d_{1}\left(a_{1} a_{9}+a_{2} a_{8}\right)-\mu \gamma_{2} a_{2} d_{1} a_{9}-\alpha d_{2} a_{25}, \\
g_{5} & =\beta \mu f_{5}+\theta\left(a_{1} b_{2}+a_{2} b_{1}\right)+\mu a_{2} b_{2}+\theta(\mu+\alpha) a_{2} b_{2}-\theta \gamma_{2} a_{2} d_{1} a_{9}-\alpha d_{2} a_{26}, \\
g_{6} & =\theta a_{2} b_{2} .
\end{aligned}
$$

The positive endemic equilibria $Q^{* *}$ are obtained by solving for $\lambda^{* *}$ from the sixth equation (A.9) and substituting the result (positive values of $\lambda^{* *}$ ) into the expressions of the state variables of model system (3.17) at the steady state defined as in Eqs. (A.3) and (A.5). Clearly, it can be shown that the coefficient $g_{0}$ of (A.9) is positive or negative whenever $\mathcal{R}_{0}$ is greater or less than unity, respectively. Thus, the number of possible real roots of the polynomial (A.9) can have depends on the signs of the coefficients $g_{6}, g_{5}, g_{4}, g_{3}, g_{2}, g_{1}$ and $g_{0}$. This can be analyzed using the Descartes Rule of Signs on the polynomial $h\left(\lambda^{* *}\right)=g_{6}\left(\lambda^{* *}\right)^{6}+g_{5}\left(\lambda^{* *}\right)^{5}+g_{4}\left(\lambda^{* *}\right)^{4}+g_{3}\left(\lambda^{* *}\right)^{3}+g_{2}\left(\lambda^{* *}\right)^{2}+g_{1} \lambda^{* *}+g_{0}$.

Using the Descarte Rule of Signs, one can conclude that the TB model (2.3) or (3.17) 
1. could have a unique endemic equilibrium whenever $\mathcal{R}_{0}>1$;

2. could have more than one endemic equilibrium whenever $\mathcal{R}_{0}>1$;

3. could have a unique endemic equilibrium whenever $\mathcal{R}_{0}<1$;

4. could have one or more endemic equilibria whenever $\mathcal{R}_{0}<1$.

Acknowledgements. Samuel Bowong acknowledges the financial support of the ICTP in Trieste-Italy under the Associate Federation Scheme.

\section{References}

[1] Global tuberculosis report. Geneva: World Health Organization; Available from: http://whqlibdoc.who.int/ publications/2010/9789241564069-eng.pdf [accessed 25 March 2011], 2010.

[2] C. Dye, B.G. Williams. The population dynamics and control of tuberculosis. Science 328 (2010), 856-861.

[3] K. Lönnroth, K.G. Castro, J.M. Chakaya, L.S. Chauhan, K. Floyd. Tuberculosis control and elimination 2010-50: cure, care, and social development. Lancet 375 (2010), 1814-1829.

[4] J. Stephenson. TB progress slowing. JAMA 299 (2008), 1764-1771, doi: 10.1001/jama.299.15.1764-b.

[5] The World Health Report, Diabetes, Fact sheet $N^{0} 312$, http://www.who.int/mediacentre/factsheets/fs312/en/, Jan. 2011.

[6] American Diabetes Association. Diagnosis and classification of diabetes mellitus. Diabetes Care 32 (2008), 62-67.

[7] Diabetes atlas. Brussels, International Diabetes Federation; Available from: http://www.diabetesatlas.org/ 2010, [accessed 25 March 2011].

[8] C. Dye. Global epidemiology of tuberculosis. Lancet 367 (2006), 938-940, doi:10.1016/S0140-6736(06)68384-0 PMID:16546542.

[9] P.N. Oscarsson, H. Silwer. Incidence of pulmonary tuberculosis among diabetics. Acta Med. Scand. 335 (1958), $23-48$.

[10] H.F. Root. The association of diabetes and tuberculosis. N. Engl. J. Med. 210 (1934), 1-13. doi:10.1056/NEJM193401042100101.

[11] S.J. Kim, Y.P. Hong, W.J. Lew, S.C. Yang, E.G. Lee. Incidence of pulmonary tuberculosis among diabetics. Tuber. Lung Dis. 76 (1995), 529-533. doi:10.1016/0962-8479(95)90529-4 PMID:8593374.

[12] M.A. Mori, G. Leonardson, T.K. Welty. The benefits of isoniazid chemoprophylaxis and risk factors for tuberculosis among Oglala Sioux Indians. Arch. Intern. Med. 152 (1992), 547-50, doi:10.1001/archinte.152.3.547 PMID:1546917

[13] F. Mugusi, A.B. Swai, K.G. Alberti, D.G. McLarty. Increased prevalence of diabetes mellitus in patients with pulmonary tuberculosis in Tanzania. Tubercle 71 (1990), 271-276, doi:10.1016/0041-3879(90)90040-F PMID:2267680

[14] A. Pablos-Mèndez, J. Blustein, C.A. Knirsch. The role of diabetes mellitus in the higher prevalence of tuberculosis among Hispanics. Am J Public Health 87 (1997), 574-579, doi:10.2105/AJPH.87.4.574 PMID:9146434

[15] C.Y. Jeon, M.B. Murray. Diabetes mellitus increases the risk of active tuberculosis: a systematic review of 13 observational studies. PLoS Med 5 (2008), 152. doi:10.1371/journal.pmed.0050152 PMID:18630984

[16] I.B. Restrepo, A.J. Camerlin, M.H. Rahbar, W. Wang, M.A. Restrepo, I. Zarate, F. Mora-Guzmán, J. G. Crespo-Solis, J. Briggs, J. B. McCormicka, S. P. Fisher-Hocha. Cross-sectional assessment reveals high diabetes prevalence among newly-diagnosed tuberculosis cases. Bull. World Health. Organ. 89 (2011), 352-359, doi:10.2471/BLT.10.085738 359.

[17] M.M. McMahon, R.B. Bistrian. Host defenses and susceptibility to infection in patients with diabetes mellitus. Infect. Dis. Clin. North Am. 9 (1995), 1-10.

[18] H. Koziel, M.J. Koziel. Pulmonary complications of diabetes mellitus. Infect. Dis. Clin. North Am. 9 (1995), 67-72.

[19] K. Tsukaguchi, H. Okamura, M. Ikuno. The relation between diabetes mellitus and IFN-gamma, IL-12 and IL-IO production by CD4+T cells and monocytcs in patients with pulmonary tuberculosis. Kekkaku, 72 (1997), 617-628.

[20] M.A. Karuchunskn, V. Gcrgcrt, O.B. lakovlcva. Specific features of cellular immunity of pulmonary tuberculosis in patients with diabetes mellitus. Problem Tnberk 6 (1997), 59-63.

[21] C.T. Yu, C.H. Wang, T.J. Huang. Relation of bronchoalvcolar lavagc T lymphocyte subpopulations to rate of regression of active pulmonary tuberculosis. Thorux 50 (1995), 86-93.

[22] A. Guptan, A. Shah. Tuberculosis and diabetes: An appraisa. Ind. J. Tub. 47 (2000), 3-12.

[23] C.R. Stevenson, N.G. Forouhi, G.C. Rogli, B.G. Williams, J.A. Lauer, C. Dye, N. Unwin. Diabetes and tuberculosis: the impact of the diabetes epidemic on tuberculosis incidence. BMC Public Health 7 (2007), 234-242, doi:10.1186/14712458-7-234.

[24] M.B. Murray, C.Y. Jeon. Diabetes mellitus increases the risk of active tuberculosis: A systematic review of 13 observational studies. PLoS Medicine 5 (2008), 7-15, e152.doi:10.1371/journal.pmed.0050152.

[25] C. Dye, B. Bourdin Trunz, K, Lönnroth, G., Roglic, B.G. Williams. Nutrition, diabetes and tuberculosis in the epidemiological transition. PLoS One, 6(6): e21161., 2011, doi: 10.1371/journal.pone.0021161

[26] K.E. Dooley, R.E. Chaisson. Tuberculosis and diabetes mellitus: convergence of two epidemics. Lancet Infect. Dis. 9 (2009), 737-46.

[27] C. Castillo-Chavez, B. Song.Dynamical models of tuberculosis and their applications. Math. Biosci. Eng. 1 (2004), 361-404. 
[28] C.P. Bhunu, W. Garira, Z. Mukandavire, M. Zimba. Tuberculosis transmission model with chemoprophylaxis and treatment. Bull. Math. Biol. 70 (2008), 1163-1191.

[29] T. Cohen, C. Colijn, B. Finklea, M. Murray. Exogenous re-infection and the dynamics of tuberculosis epidemics: local effects in a network model of transmission. J. R. Soc. Interface 4 (2007), 523-531.

[30] S. Bowong, J.J. Tewa. Mathematical analysis of a tuberculosis model with differential infectivity. Com. Nonl. Sci. Num. Sim. 14 ( 2009), 4010-4021.

[31] Z. Feng, C.C. Chavez, A. F. Capurro. A model for tuberculosis with exogenous reinfection. Theor. Popul. Biol. 57 (2000), 235-247.

[32] B.M. Murphy, B.H. Singer, S. Anderson, S. Kirschner, Comparing epidemic tuberculosis in demographically distinct heterogeneous populations. Math. Biosci. 180 (2002), 161-185.

[33] R.W. Shafer, S.P. Singh, C. Larkin, P.M. Small. Exogenous reinfection with multidrug-resistant Mycobacterium tuberculosis in an immunocompetent patient. Tuberc. Lung Dis. 76 (1995), 575-577.

[34] K. Styblo, J. Meijer, I. Sutherland. The transmission of tubercle bacilli: its trend in a human population. Bull. Int. Union Tuberc. 42 (1969), 5-104.

[35] National Institute of Statistics, Evolution des systèmes statistiques nationaux, expérience du Cameroun, in The National Institute of Statistics report, J. Tedou (ed). New-York, (2010), 1-18.

[36] National Comittee of Fight Against Tuberculosis, Guide du personnel de la santé, in The Ministry of Public Health report, Ministére de la Santé Publique (ed), Yaoundé-Cameroon: CEPER Press (2010), 1-110.

[37] N. Bacaër, R. Ouifki, C. Pretorius, R. Wood, B. Williams. Modeling the joint epidemics of TB and HIV in a South African township. J. Math. Biol. 57 (2008), 557-593, DOI 10.1007/s00285-008-0177-z.

[38] C. Dye, B.G. William. Criteria for the control of drug resistant tuberculosis. Proc. Natl. Acad. Sci. USA 97 (2000), $8180-8185$.

[39] T. Cohen, M. Murray, Modeling epidemics of multidrug-resistant M. tuberculosis of heterogeneous fitness, Nature Publishing Group,http://www.nature.com/naturemedicine, (2004).

[40] G. Birkhoff, G.C. Rota, Ordinary Differential Equations. 4th edition, John Wiley and Sons, Inc., New York., 1989.

[41] A. Berman, R.J. Plemmons, Nonnegative matrices in the mathematical sciences, SIAM., 1994.

[42] J.A. Jacquez, C.P. Simon, Qualitative theory of compartmental systems. SIAM Rev. 35 (1993), 43-79.

[43] P. van den Driessche, J. Watmough. Reproduction numbers and sub-threshold endemic equilibria for compartmental models of disease transmission. Math. Bios. 180 (2002), 29-28.

[44] C. Castillo-Chavez, Z. Feng, D. Xu, On the computation of $\mathcal{R}_{0}$ and its role on global stability. math.la.asu.edu/chavez/2002/JB276.pdf, 2002.

[45] J. Carr, Applications Centre Manifold theory. Springer-Verlag, New York, 1981.

[46] O. Sharomi, A.B. Gumel. Curtailing smoking dynamics: a mathematical modeling approach. Appl. Math. Comput. 19 (2008), 475-499.

[47] N. Chitnis, J.M. Hyman, J.M. Cushing. Determining important parameters in the spread of malaria through the sensitivity analysis of a mathematical model. Bull. Math. Biol. 70 (2008), 1272-1296.

[48] C.R. Stevenson, J.A. Critchley, N.G. Forouhi, G. Roglic, B.G. Williams. Diabetes and the risk of tuberculosis: a neglected threat to public health? Chronic Illness 3 (2007), 228-245,

[49] C.K.W. Gavin Koh, W.J. Wiersinga. Tuberculosis and Diabetes Mellitus: Convergence of Two Epidemics: F1000 Ranking: "Changes Clinical Behavior". Lancet Infect. Dis. 9 (2009), 737-46.

[50] C. Dye, B.B. Trunz, K. Lonnroth, G. Roglic, B.G. Williams. Nutrition, Diabetes and Tuberculosis in the Epidemiological Transition. PLoS ONE 6(6): e21161. doi:10.1371/journal.pone.0021161. 

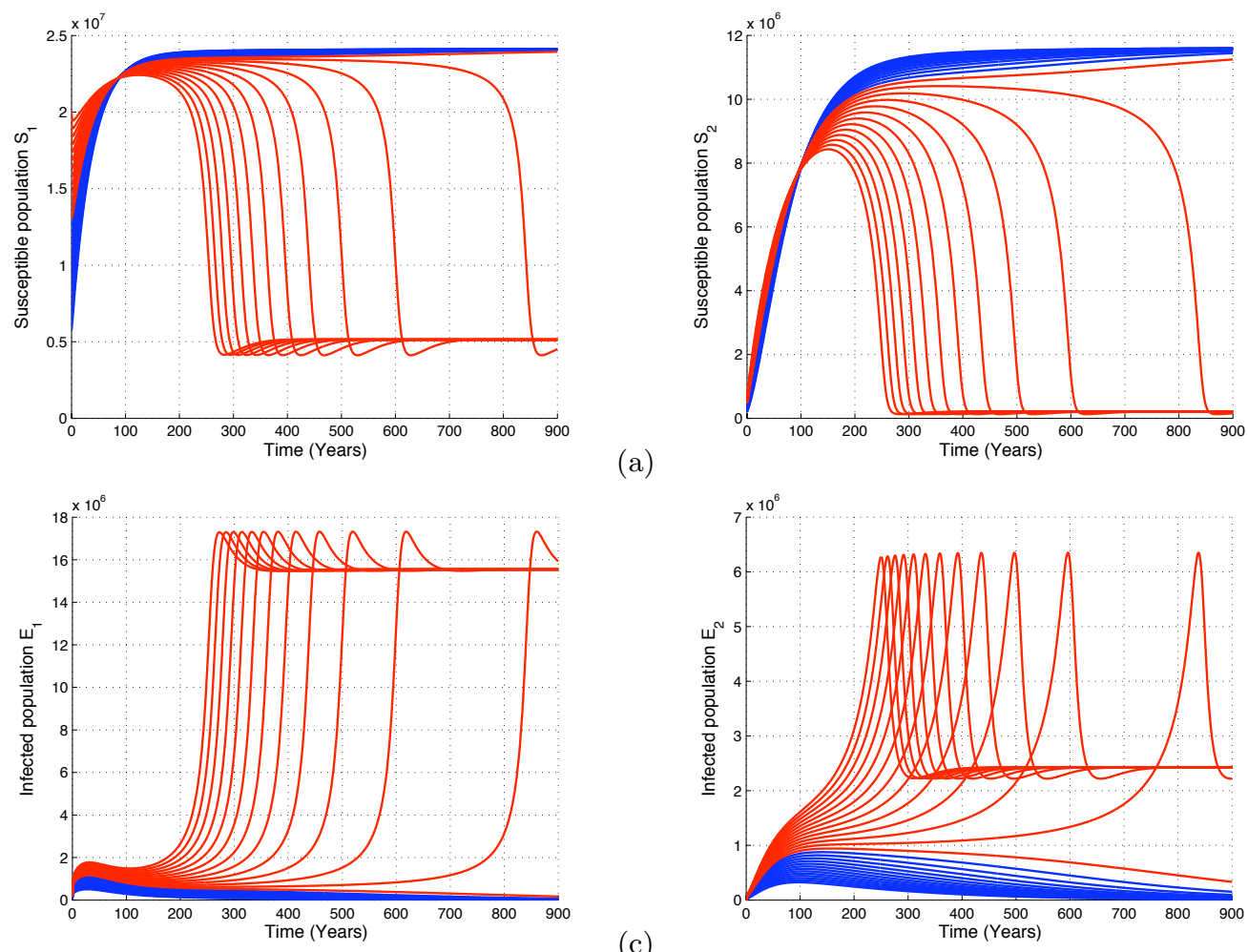

(a)

(c)
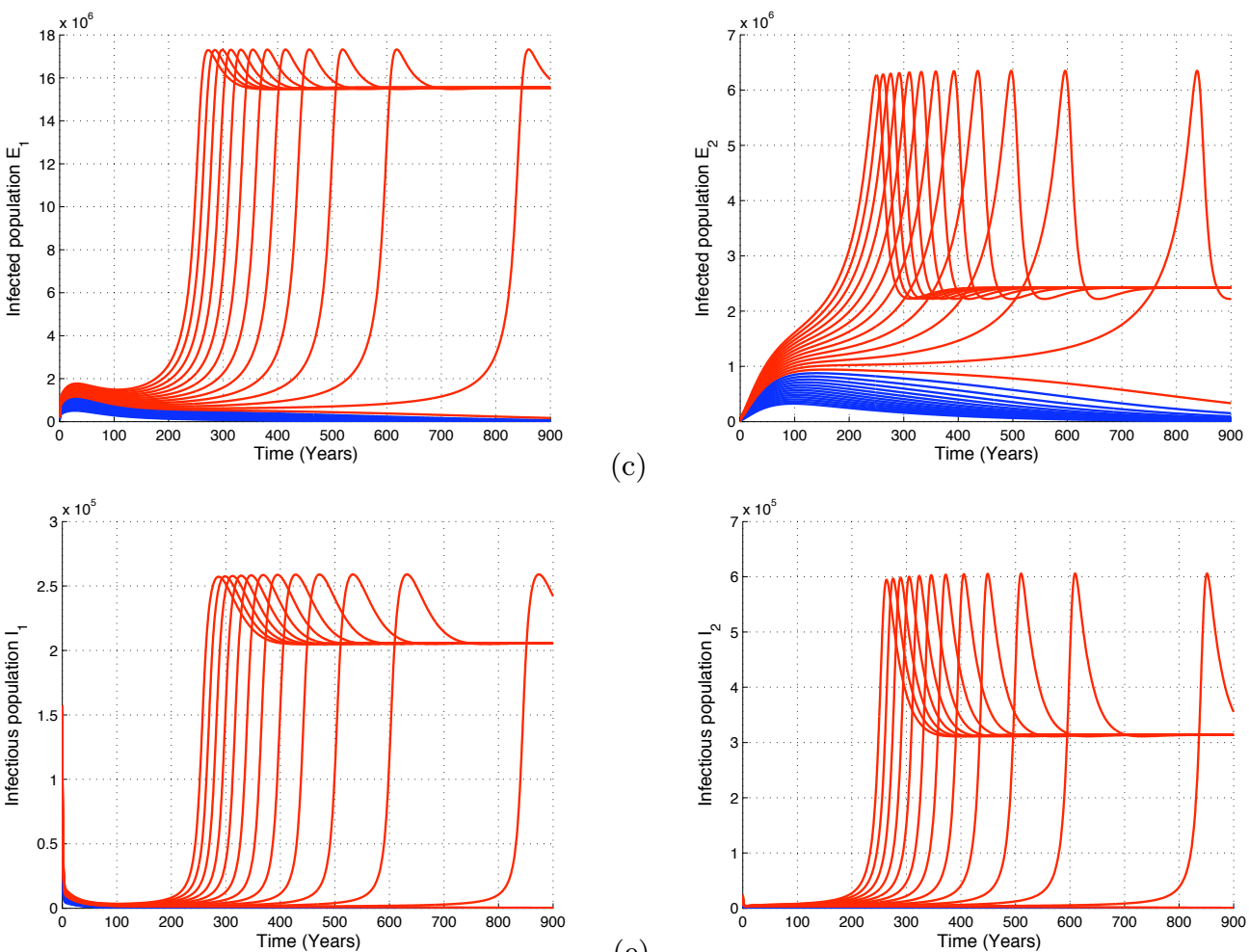

(e)
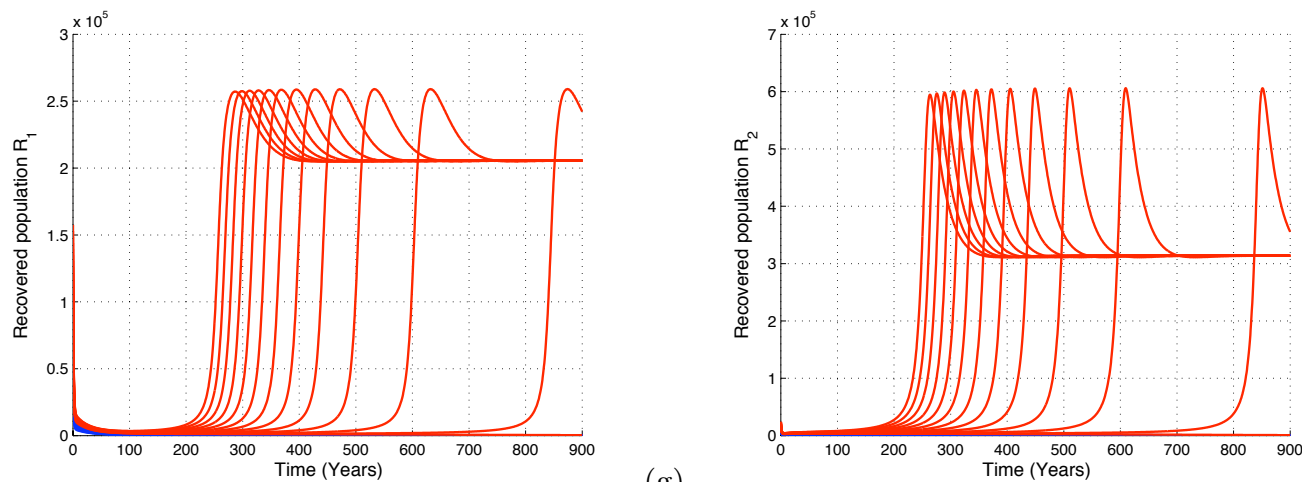

$(\mathrm{g})$

Figure 5. Simulation of model system (2.3) using various initial conditions when $\beta=$ 7 and $\sigma_{1}=0.05$ (so that $\mathcal{R}_{0}=0.8597$ ). Time series of (a) Susceptible population $S_{1}$, (b) Susceptible population $S_{2}$, (c) Latently infected population $E_{1}$, (d) Latently infected population $E_{2}$, (e) Infectious poputation $I_{1}$, (f) Infectious population $I_{2}$ and (g) Recovered population $R_{1}$, (h) Recovered population $R_{2}$. All other parameters are as in Table 1. 

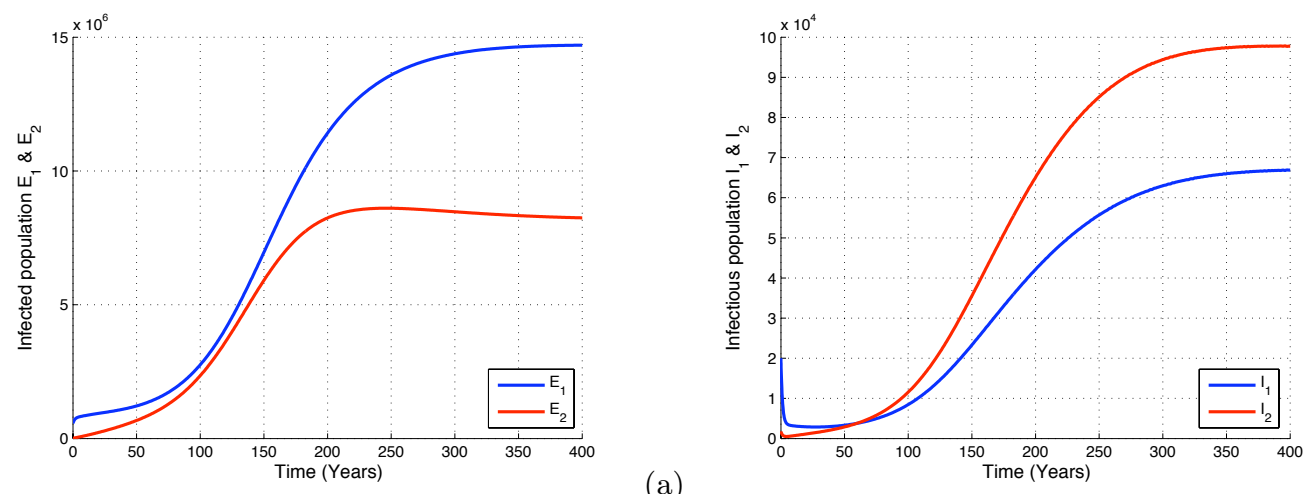

FiguRE 6. Simulation results showing the general trends of model system (2.3) when $\beta=$ 10 (so that $\mathcal{R}_{0}=1.7194$ ). (a) Non-diabetics and diabetics latently infected individuals and (b) Non-diabetics and diabetics infectious. All other parameters are as in Table 1.

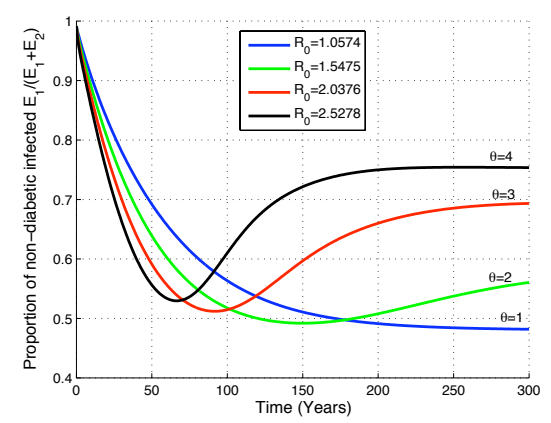

(a)
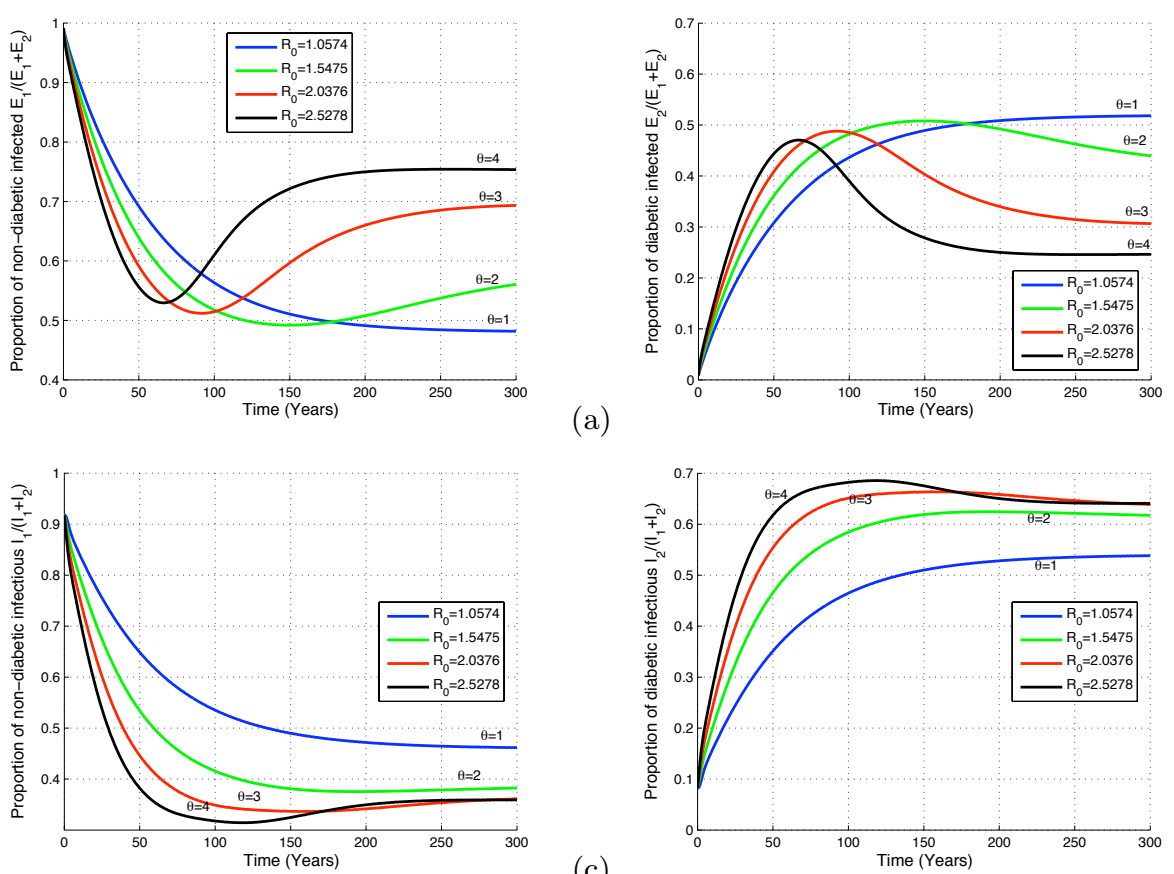

(c)

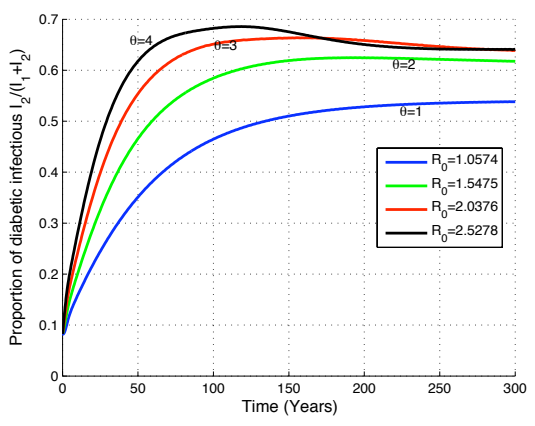

(b)

(d)

FiguRE 7. Simulation results showing the effect of increasing the susceptibility to TB du to DM $\theta$ when $\beta=9$ (so that $\mathcal{R}_{0}>1$ ). All other parameters are as in Table 1 . 


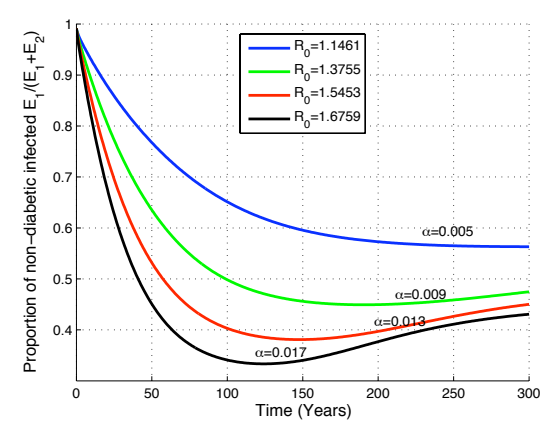

(a)
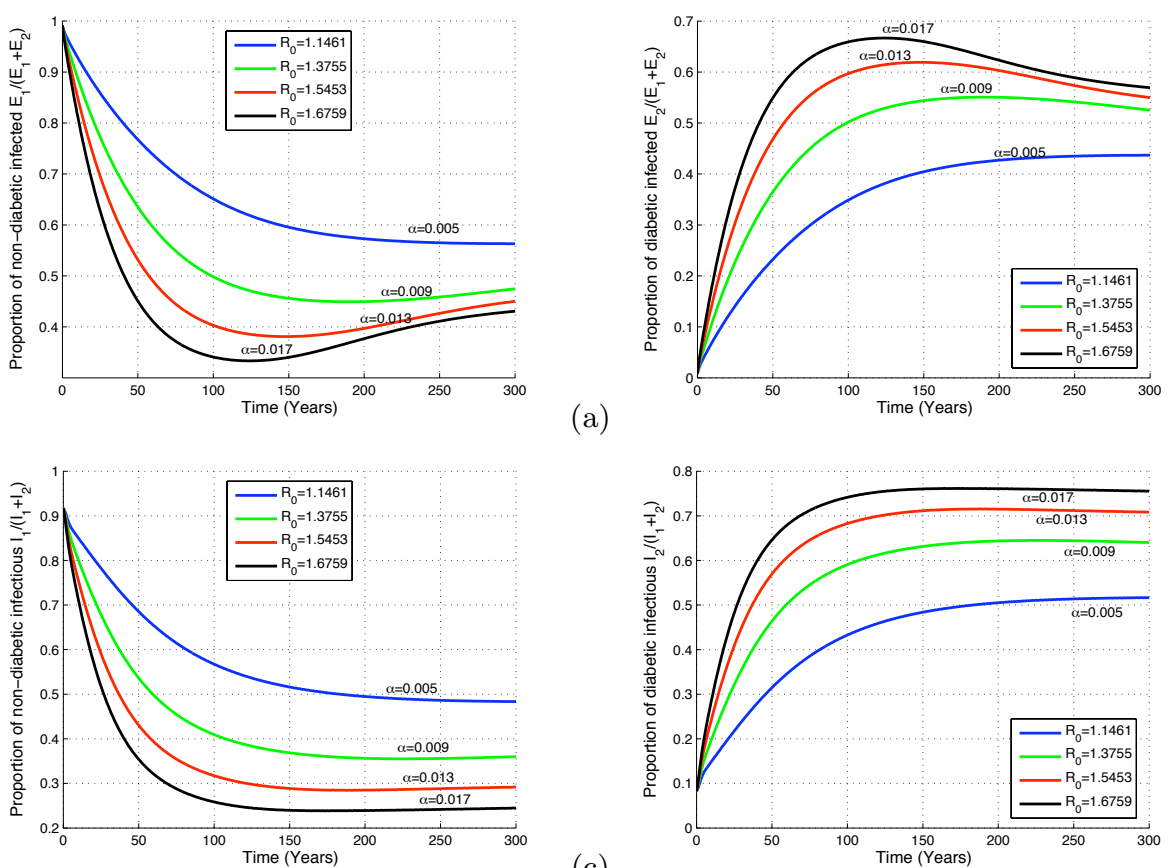

(c)

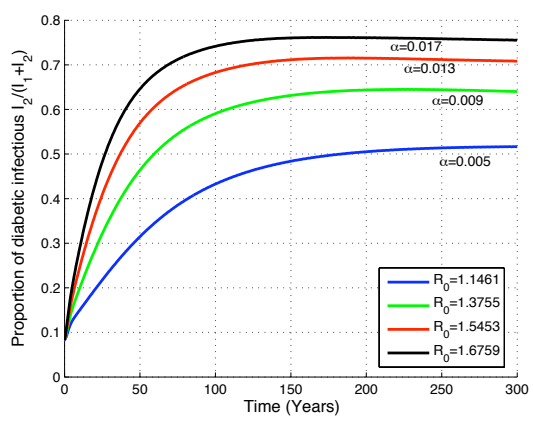

(b)

FigURE 8 . Simulation results showing the effect of increasing the rate of being diabetics $\alpha$ when $\beta=8$ (so that $\mathcal{R}_{0}>1$ ). All other parameter values as given in Table 1 .

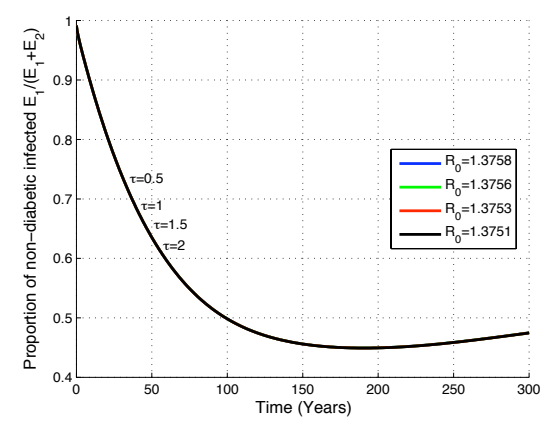

(a)
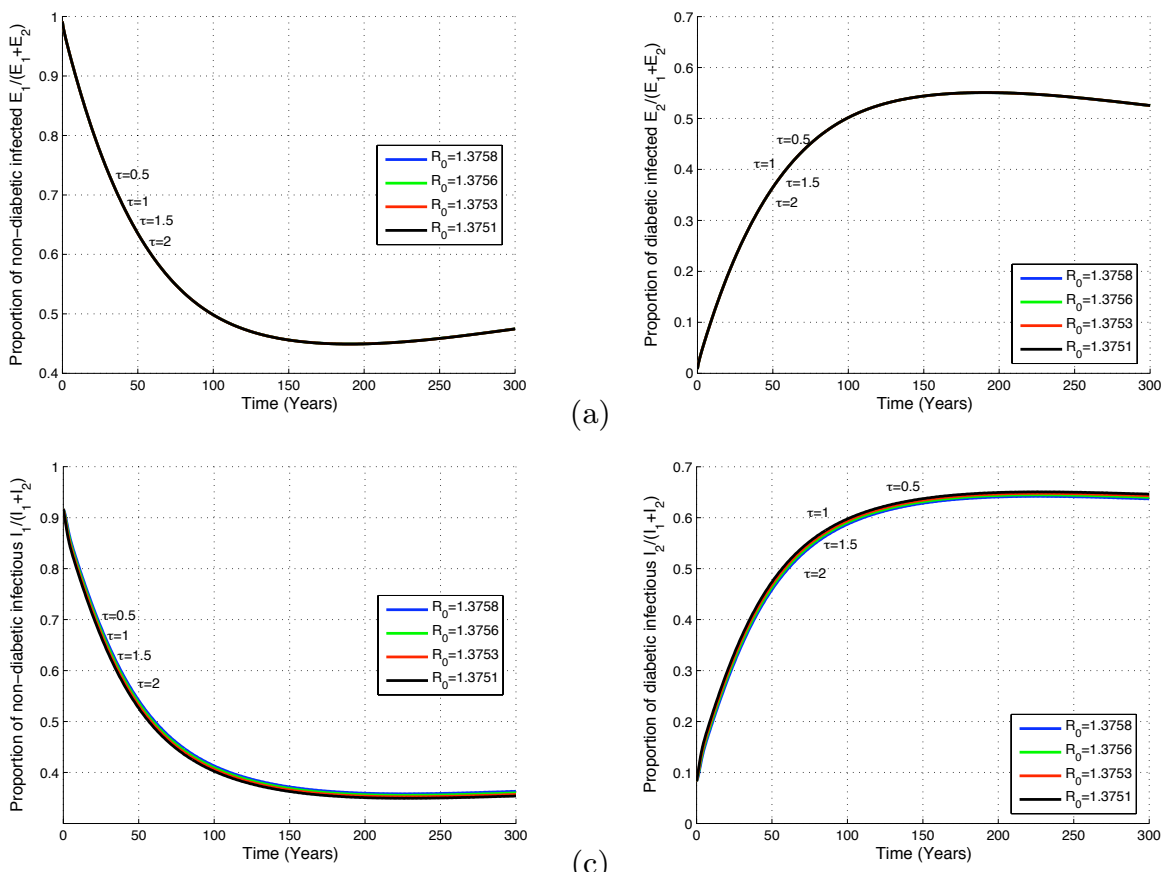

(c)

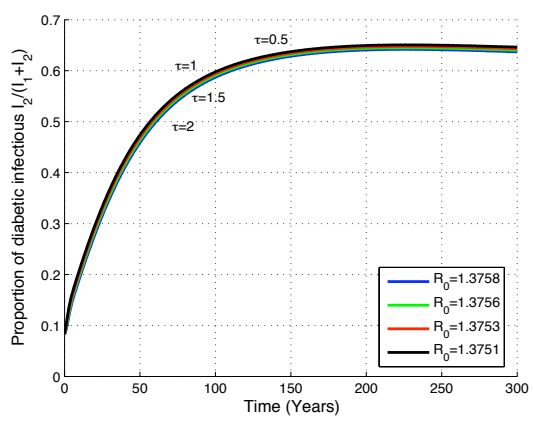

(b)

FIGURE 9. Simulation results showing the effect of increasing the modification parameter $\tau$ when $\beta=8$ (so that $\mathcal{R}_{0}>1$ ). All other parameter values as given in Table 1 . 


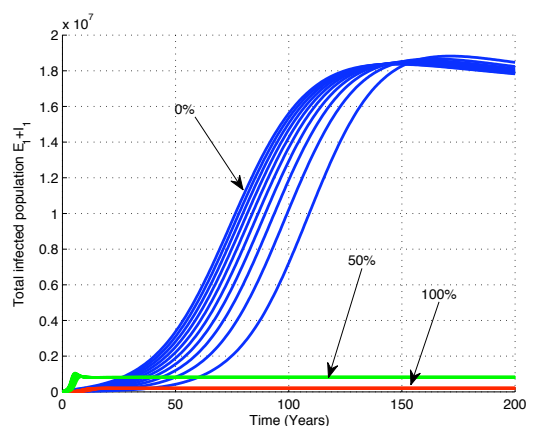

(a)
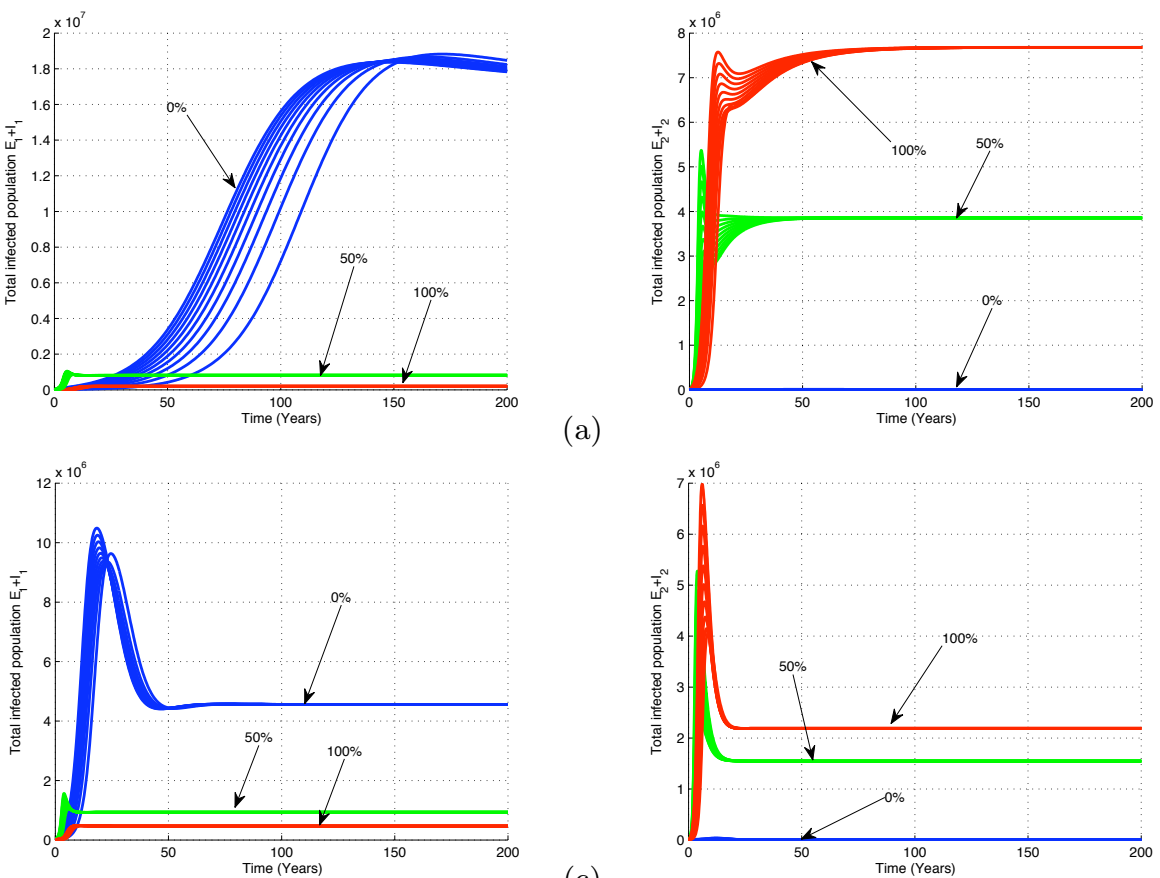

(b)

(c)

(d)

FiguRE 10. Simulations of model system (2.3) showing the effects of varying the percentage of diabetics inside the population on TB infected cases in the absence and presence of treatment. Parameter values used are in Table 1. 